\title{
An Improved Model for Polyether Production from 1,3-Propanediol
}

\author{
Anh-Duong Dieu Vo, Aly Elraghy, Rupert E. Spence, and Kimberley B. McAuley*
}

\begin{abstract}
A dynamic mathematical model is developed for production of Cerenol polyether from 1,3-propanediol in a batch reactor system. The model accounts for polycondensation reactions and side reactions in the liquid phase and for mass transfer of volatile species to the vapor. Parameters are estimated using measured liquid-phase concentrations of monomer, oligomers, water, and end groups as well as the mass and composition of condensate collected from the overhead condenser system. The proposed model uses novel probability factors to keep the model equations relatively simple while accounting for the complex influence of superacid catalyst on reaction rates. The model is a significant advance over previous Cerenol models because it better accounts for mass-transfer rates and for the dynamic behavior of the condenser. In addition, the proposed model accounts for the inhibitory influence of water on polycondensation kinetics due to hydration of hydroxyl ends. The model equations and parameter estimates provide a substantial improvement in fit to the data, especially for long reaction times and high catalyst levels, resulting in a $97 \%$ reduction in the value of the weighted least squared objective function compared to equations and parameters from a previous model.
\end{abstract}

properties, PO3G is used in various applications including cosmetics, breathable membranes, biodegradable lubricants, architectural coatings, adhesives, and elastomeric fibers..$^{[17,18]}$

Several mathematical models have been developed to obtain improved understanding of the influence of process operating conditions on product properties and production rates during PO3G production. ${ }^{[19-23]}$ Mueller et al. developed the first fundamental PO3G model, which accounts for the influence of two different acid catalysts. ${ }^{[20]}$ Their model predicts time-varying concentrations of propanediol and water in the reactor liquid and in the vapor that is removed via nitrogen sparging. Their model also predicts number-average molecular weight and concentrations of hydroxyl and unsaturated end groups in the polymer. Their model does not include evaporation of oligomers (e.g., linear, dimer, trimer, etc.) from the reaction mixture. Although

\section{Introduction}

In recent years, bio-based poly(trimethylene) ether glycol (PO3G) has been manufactured using renewable feedstocks. ${ }^{[1-18]}$ The trademark for this class of polymers is Cerenol and the monomer is bio-based 1,3-propanediol (bio-PDO), which was commercialized by DuPont. ${ }^{[1-13]}$ The production of Cerenol occurs at relatively low temperatures and pressures in comparison to processes for petroleum-based polyether diols, making the production process more sustainable and inherently less hazardous. ${ }^{[14,15]}$ PO3G is biodegradable, has low toxicity, low viscosity, and low melting point compared to petroleum-based polyether diols. ${ }^{[14-16]}$ Because of its favorable

\author{
A.-D. Dieu Vo, A. Elraghy, Prof. K. B. McAuley \\ Department of Chemical Engineering \\ Queen's University \\ Kingston, Ontario K7K 3N6, Canada \\ E-mail: kim.mcauley@queensu.ca \\ Dr. R. E. Spence \\ E.I du Pont Canada Company \\ Research and Business Development \\ Kingston, Ontario K7L 5A5, Canada
}

The ORCID identification number(s) for the author(s) of this article can be found under https://doi.org/10.1002/mren.201900045.

DOI: 10.1002/mren.201900045
Mueller et al. report values for equilibrium constants for some of the acid-base protonation/deprotonation reactions in their model, they do not report values for any of the kinetic parameters used to obtain their model predictions.

Cui et al. extended Mueller's reaction scheme to account for formation of propanal (a side product) and for transetherification reactions that randomize the molecular weight distribution when monomer and oligomers evaporate from the liquid phase. ${ }^{[21-23]}$ They used data from the batch reactor system in Figure $1,{ }^{[21-23]}$ to estimate parameters in a series of three models with increasing levels of sophistication. As shown in Figure 1, nitrogen is bubbled through the liquid phase to remove water from the reaction mixture so that PO3G with a high degree of polymerization (DP) can be produced. A portion of the monomer (and oligomers) evaporate with the water. Samples of the reactor liquid and the condensate were collected over the course of each batch reactor run and were subsequently analyzed, providing information about reaction kinetics and evaporation rates.

The first model of Cui et al. predicts the time evolution of DP, along with concentrations of water, monomer, propanal, and unsaturated ends. Evaporation rates of water, monomer, and propanal are also predicted. Cui et al. used estimability analysis techniques ${ }^{[24,25]}$ to rank the kinetic and transport parameters in their model from most-estimable to least-estimable and used a mean-squared-error criterion ${ }^{[26,27]}$ to determine that 8 of their 


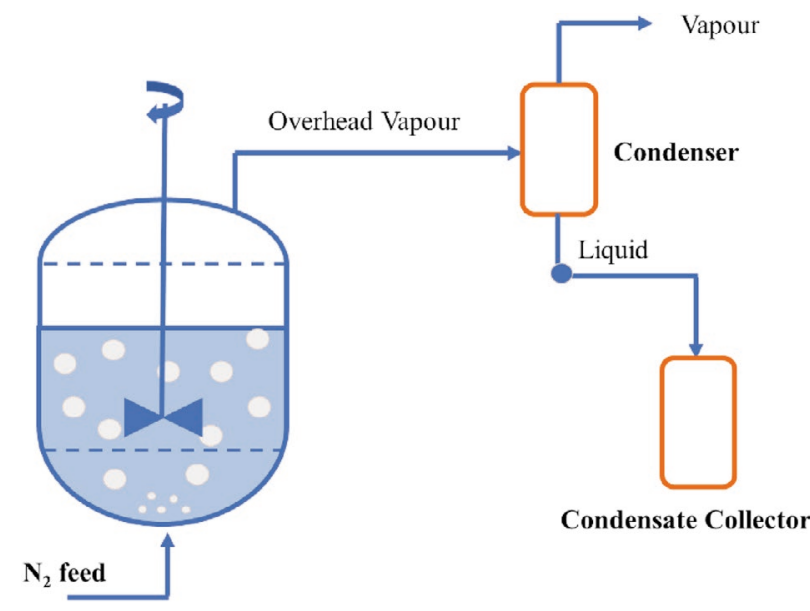

Figure 1. PO3G reactor and condenser system. ${ }^{[21-23]}$

14 model parameters could be estimated from four dynamic batch-reactor runs conducted at $180{ }^{\circ} \mathrm{C}$ using different concentrations of super-acid catalyst. ${ }^{[21]}$ Cui's first model neglected evaporation of oligomers and assumed that mass-transfer coefficients for water and monomer are constant over the duration of each batch run. Unfortunately, this model gives relatively poor predictions, especially of DP at long reaction times. ${ }^{[21]}$ Cui et al. performed a second modeling study to investigate the influence of DP on mass-transfer rates to determine whether time-varying mass-transfer coefficients could help to improve the model predictions. ${ }^{[22]} \mathrm{Cui}$ et al. showed that increases in bubble residence time due to increasing viscosity have an important influence on mass-transfer rates and extended their previous model to account for these effects. Although predictions improved somewhat, predictions of DP at long reaction times and of residual monomer concentrations in the liquid phase were unsatisfactory. ${ }^{[22]}$ A third model was developed to account for evaporation of linear oligomers (i.e., dimer to pentamer), which was neglected in previous models, even though these species make up $\approx 10 \mathrm{wt} \%$ of the condensate collected from the overhead stream shown in Figure $1 .{ }^{[23]}$ This third model resulted in better predictions of DP (which is still underpredicted at long reaction times) but provided a relatively poor fit to data for monomer concentrations in the reactor, especially at long reaction times. Underprediction of DP and overprediction of the monomer concentration at long reaction times suggest that the overall rate of polycondensation predicted by Cui's model is too low, especially during the final low-water stage of the batch reactor experiments. This problem may result from inappropriate reaction kinetics or mass-transfer expressions. Including mass transfer of oligomers in the model resulted in four additional mass-transfer parameters. ${ }^{[23]}$ Cui et al. showed that these four new parameters could be estimated from the available data, along with 11 of the 14 other model parameters. ${ }^{[23]}$ Although qualitative predictions of the linear oligomer concentrations in the reactor liquid were good, Cui et al. noted that the predicted maxima in the dimer, trimer, and tetramer concentrations occurred earlier than the maxima in the corresponding measured concentrations. Also, predicted oligomer concentrations in the reactor were too high at long times. Cui et al. suggested that discrepancies in the predictions could be because: i) simplifying assumptions were made about the overhead condenser system (i.e., no time delay in the piping and negligible liquid hold-up in the condenser); ii) mass-transfer expressions for monomer and water were developed based on restricted experimental vapor-liquid equilibrium (VLE) data; iii) there may be deficiencies in the reaction scheme used to develop the model (e.g., the formation of cyclic oligomers is neglected). ${ }^{[23]}$ In the improved model developed in this paper, we address these issues (except for cyclic-oligomer formation, which is the topic of ongoing research).

Cui's models and the model of Mueller et al. neglected the dynamics of the overhead condenser system, assuming that water, monomer, and oligomers that evaporate from the reaction mixture appear instantaneously in the liquid condensate that enters the condensate collector (see Figure 1). ${ }^{[19-23]}$ However, there is a substantial hold-up of accumulated liquid in the condenser, which introduces an additional lag in the measured condensate concentrations. Time delay associated with flow through the overhead piping may also influence the condensate concentration measurements that were used for model-fitting by Cui et al. In the current article, the model is extended to account for the mass of liquid in the condenser and for time delay, leading to two additional parameters that appear in the model. ${ }^{[28]}$

Mass-transfer expressions developed by Cui et al. rely on experimental VLE data shown in Figure 2. ${ }^{[22]}$ Figure 2a shows the vapor pressure of water, $P_{\mathrm{W}}$, plotted versus the corresponding equilibrium volume fraction of water, $F_{\mathrm{vW}}^{*}$, in molten PO3G. The dashed curve is a prediction at $180{ }^{\circ} \mathrm{C}$ from a Flory-Huggins based correlation developed by Xie. ${ }^{[16]}$ Xie's correlation was fitted using data obtained over a relatively narrow composition range. Figure $2 \mathrm{~b}$, showing the corresponding vapor pressure of monomer, $P_{\mathrm{L} 1}$, considers a wider range of volume fractions because the experiments conducted in the reactor shown in Figure 1 start with pure 1,3-propanediol at the beginning of each batch. The monomer volume fraction in the liquid decreases over time due to polymerization and evaporation. The curve in Figure $2 \mathrm{~b}$ is a cubic spline fit to VLE data for the monomer. ${ }^{[22]}$ In the current modeling study, two additional parameters are introduced to the water VLE model to obtain good fits to the dynamic reactor operating data. Cui's cubic spline expressions for monomer VLE ${ }^{[22]}$ are used without any changes.

The mass-transfer expressions developed by Cui et al. assume that partial pressures of water and monomer in the bubbles are uniform (from bubble to bubble), regardless of their vertical position within the reactor (i.e., the bubble phase is well-mixed due to recirculation and coalescence of bubbles within the reacting mixture). ${ }^{[22]}$ However, as nitrogen bubbles rise from the bottom of the reactor to the liquid surface, partial pressures of monomer and water in the bubbles may vary considerably with vertical position. Bubbles that form near the nitrogen inlet will have near-zero concentrations of water and monomer, whereas bubbles that emerge at the top surface of the liquid will tend to have higher concentrations. The amount of backmixing and coalescence of bubbles within the reactor is not well understood and could be small. To deal with this uncertainty, an adjustable bubble-backmixing parameter is included in the model proposed in the current article.

In the reaction mechanisms used in the models of Cui et al. and Mueller et al., it is assumed that the rate constant (and 

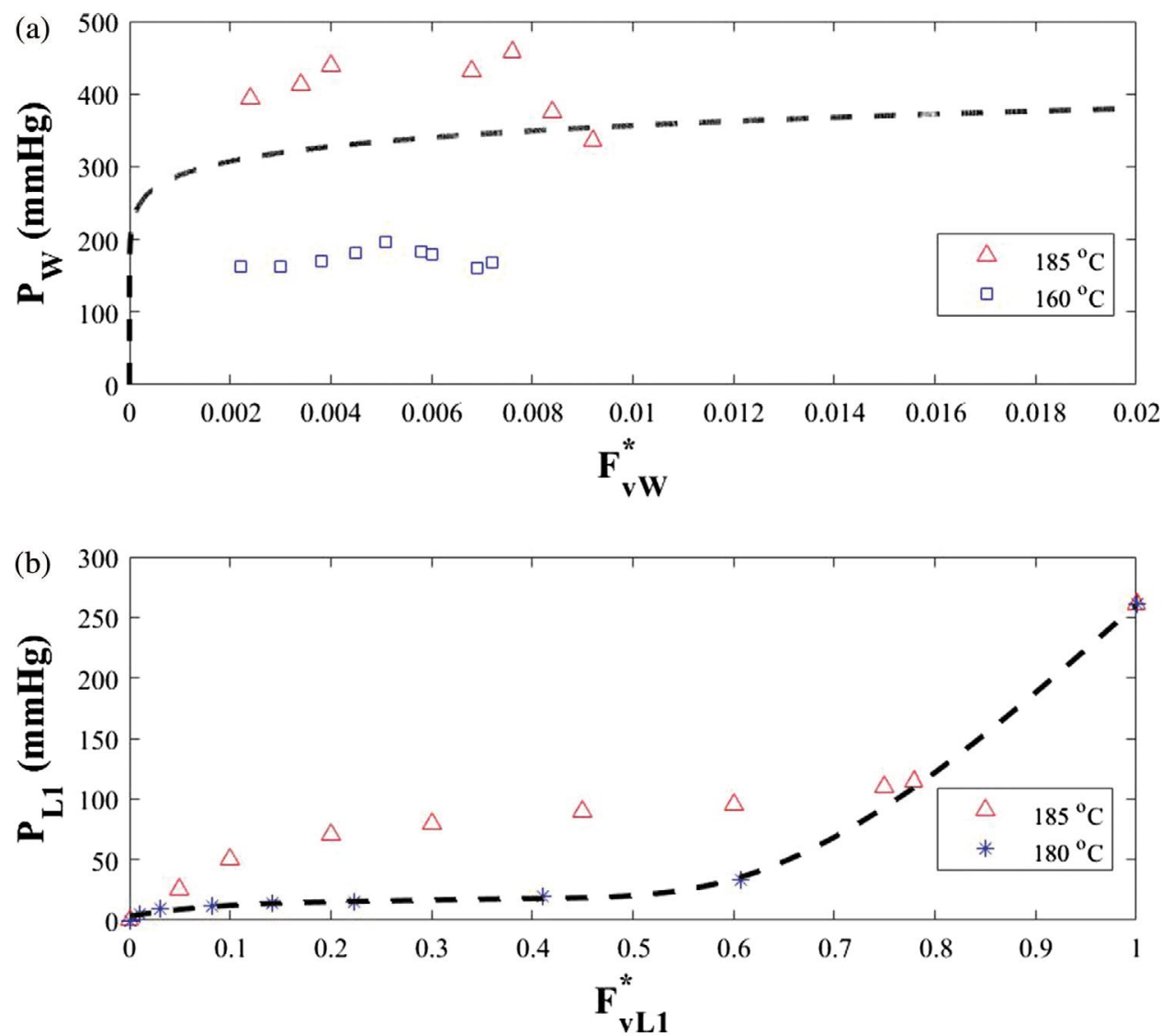

Figure 2. a,b) Vapor-liquid equilibrium correlations for water and monomer at $180^{\circ} \mathrm{C}$ and experimental data at several temperatures. ${ }^{[16,22]}$

equilibrium constant) for the main polycondensation reaction is independent of the water concentration. ${ }^{[19-23]}$ However, this assumption may not be valid and may be an important source of the discrepancies between Cui's model predictions and the dynamic PO3G data. The research group of Yin et al. showed that water has an important inhibitory effect on the rate of etherification reactions. ${ }^{[29-31]}$ For example, Yin et al. investigated the etherification of ethanol and tert-butyl alcohol to produce ethyl tert-butyl ether, with and without added water, in the presence of an acid catalyst. ${ }^{29]}$ They found that initial reaction rates without added water were about 15 times higher (at $323 \mathrm{~K}$ ) than corresponding reaction rates in the presence of water at a concentration of $12 \mathrm{wt} \% .{ }^{[29]}$ In a follow-up study, Yang produced tert-amyl methyl ether from methanol and tert-amyl alcohol and found that this etherification reaction was also greatly inhibited by added water. ${ }^{[30,31]}$ A similar inhibition effect of water has been found for esterification ${ }^{[32,33]}$ and amidation reactions and has been incorporated in a recent nylon copolymerization model. ${ }^{[3]}$ Several empirical correlations have been developed to account for the inhibiting effect of water on esterification and etherification reaction rates. For example, Okamoto ${ }^{[32]}$ proposed the following expression

$k=\frac{k_{0}^{\prime}}{1+a[W]}$ to obtain the water-dependent reaction rate constant $k$ for esterification, based on a water-free rate constant $k_{0}^{\prime}$ and the water concentration [W]. The fitted parameter $a$ is temperature-dependent.

Yin and Yang ${ }^{[30-32]}$ modified the denominator of Okamato's expression and used it to successfully predict rates of etherification reactions using

$k=\frac{k_{0}^{\prime}}{1+a[W]^{2}}$

Later, Liu investigated the effect of water on acid-catalyzed esterification of acetic acid and methanol and determined that $k \propto[\mathrm{W}]^{-0.83} \cdot{ }^{[33]}$ Unfortunately, Liu's reaction rate equation is not applicable at an initial batch reactor condition where the concentration of water is zero, but it can be extended to a more generally applicable form

$k=\frac{k_{0}^{\prime}}{1+a[W]^{b}}$

where $-b$ is the estimated reaction order of water when [W] is large.

The main goals of the current modeling study are to extend Cui's PO3G model to account for: i) liquid hold-up and time delay in the condenser system, ii) uncertainties in water VLE 
and bubble circulation behavior, and iii) possible inhibitory effects of water on the rate of polycondensation. In addition, Cui's reaction scheme is simplified to remove inestimable parameters related to several side reactions. The revised model is then used to reestimate the model parameters, providing a substantial improvement in the fit to the data.

The remainder of the paper is organized as follows. The available kinetic data are described, followed by the revised reaction scheme and the corresponding model equations. Note that these new dynamic mass-balance equations are an important advance over the balances developed by Cui et al., because they incorporate probability factors that greatly simplify the equation structure. ${ }^{[34]}$ Results of parameter estimates and model predictions are then shown and discussed. Finally, recommendations are made to further improve the model (e.g., by including the formation of cyclic oligomers).

\section{Kinetic Data}

Industrial data were obtained from the batch reactor system shown schematically in Figure $1^{[21-23]}$ In the dynamic experiments, an initial charge of bio-PDO was fed to the reactor and maintained at a desired temperature of $180{ }^{\circ} \mathrm{C}$. Agitation and sparging with $\mathrm{N}_{2}$ was established before adding a super-acid catalyst (Triflic acid) to start the reaction. As the polycondensation reactions proceed, oligomers are produced and accumulate in the liquid phase. Water, monomer, and oligomers diffuse from the liquid into nitrogen bubbles which exit the liquid into the reactor headspace. Samples of the liquid in the reactor and the condensate were removed at selected times for analysis. Four experimental runs were conducted using initial catalyst concentrations between $0.10 \%$ and $0.25 \%$ by weight. Measurements available from polymer melt include:

1. Concentration of monomer (ppm by weight);

2. Concentration of water (wt\%);

3. Concentration of linear oligomers (i.e., dimer to heptamer) (ppm by weight);

4. Concentration of cyclic oligomers (i.e., dimer to octamer) (ppm by weight);

5. Unsaturated end group concentration $\left(\mathrm{mmol} \mathrm{kg}^{-1}\right)$.

Measurements available from the condensate include:

1. Water concentration (wt\% of overall condensate);

2. Monomer concentration (ppm by weight);

3. Linear oligomers (i.e., dimer to pentamer) (ppm by weight);

4. Cyclic oligomers (i.e., dimer to heptamer) (ppm by weight);

5. Total mass of collected condensate (g).

Note that concentrations in the condensate are reported in different units than were used by Cui et al. (previously in mol\%). Also, data for the total mass of collected condensate are used for the first time in the current parameter estimation study. Hydroxyl end-group data for the molten polymer used by Cui et al. to compute experimental DP values in previous modeling and parameter estimation studies ${ }^{[21-23]}$ are excluded from the current study because a recent analysis reveals inconsistencies with the oligomer concentration data. Details are provided elsewhere. ${ }^{[28]}$

\section{Model Development}

Table 1 shows the updated reaction mechanism used in the model. The left-hand column provides structural information, showing the polymer end groups and ether links, whereas the right-hand column shows the same reactions in terms of symbols used in the model equations. Hydroxyl ends are denoted as $O$, ether links as $E$, unsaturated ends as $U$, secondary carbocation ends as $C_{\mathrm{s}}$, and water as $W$. The subscript $\mathrm{p}$ indicates that a functional group or species is protonated. Reaction (1.1) shows the equilibrium for protons exchanging between hydroxyl ends and water with $K_{1}$ as the equilibrium constant. Cui's models also considered equilibrium protonation of ether functional groups, which is neglected in the proposed simplified model. As shown in the Supporting Information, this assumption is appropriate because Cui's model equations and parameter estimates indicate that the concentration of protonated ether links is negligible compared to the concentrations of protonated hydroxyl ends and protonated water.

Reaction (1.2) is the main polycondensation reaction, which consumes hydroxyl ends, with forward rate constant $k_{2}$ $\left(\mathrm{kg} \mathrm{mmol}^{-1} \mathrm{~h}^{-1}\right)$ and reverse rate constant $k_{2 \mathrm{r}}\left(\mathrm{kg} \mathrm{mmol}^{-1} \mathrm{~h}^{-1}\right)$. As indicated in Assumption 2.1 in Table 2, the polycondensation reaction requires one of the participating hydroxyl ends to be protonated. The reverse (hydrolysis) reaction is also acid catalyzed (i.e., it involves protonated water). Side reactions (1.3)-(1.5) produce unsaturated ends and secondary carbocation ends where $k_{3}\left(\mathrm{~h}^{-1}\right)$ is the rate constant for the end degradation reaction, $k_{4}\left(\mathrm{~kg} \mathrm{mmol}{ }^{-1} \mathrm{~h}^{-1}\right)$ is the rate constant for protonation of a terminal double bond, and $k_{5}\left(\mathrm{~h}^{-1}\right)$ is the rate constant for formation of a secondary carbocation end from a protonated hydroxyl end. Reactions (1.6) and (1.7) account for formation of propanal (denoted by AD), which is observed experimentally. ${ }^{[21-23]}$ It is assumed that reversible Reaction (1.6) is sufficiently fast to be in equilibrium (Assumption 2.8). Note that Reactions (1.6) and (1.7) are shown separately in Table 1, but we treat the protonated secondary hydroxyl end $O_{\mathrm{sp}}$ as a short-lived intermediate (Assumption 2.8) that does not accumulate in the reactor system so that the forward rate constant for Reactions (1.6) and (1.7) combined is $k_{7} K_{6}\left(\mathrm{~h}^{-1}\right)$. In Cui's earlier models, these two reactions were treated separately, but an estimability analysis revealed that parameter $K_{6}$ cannot be estimated independently from $k_{7}$ using the available industrial data. As a result, we simplified the model to contain the lumped parameter $k_{7} K_{6}$. Reaction (1.8) accounts for transetherification between oligomeric and polymer chains. The transetherification reaction is included in the model because it influences the rate at which linear oligomers are regenerated from long polymer chains to replenish oligomer molecules that evaporate from the reaction mixture. We assume that transetherification occurs between protonated hydroxyl groups and ether links. Cui's models also included transetherification involving unprotonated hydroxyl ends and protonated ether links, but based on the rate constants estimated by Cui et al., the rate of this alternative reaction is much slower than Reaction (1.8) and can be ignored.

Table 2 lists the assumptions used to develop the ordinary differential equations (ODEs) in Table 3. Assumptions 2.1 to 2.6 were made previously by Cui et al. ${ }^{[21-23]}$ Assumptions 2.7 
Table 1. Simplified mechanism for $\mathrm{PO} 3 \mathrm{G}$ production in batch reactor. ${ }^{[21-23]}$

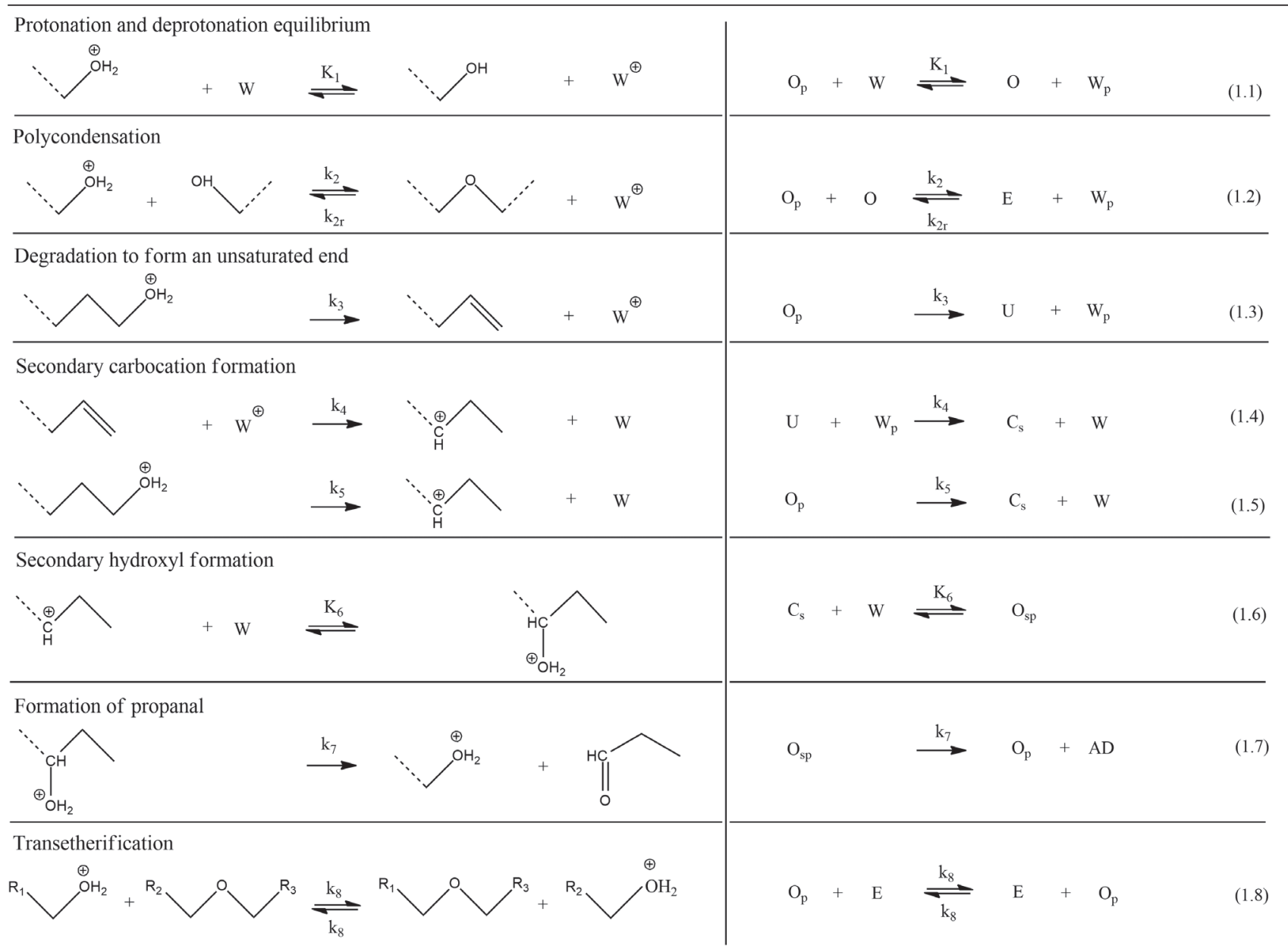

to 2.13 are new assumptions justified via simulation studies (shown elsewhere) involving Cui's model. ${ }^{[28]}$ Assumptions 2.7 to 2.11 reduce the number of material-balance equations and kinetic parameters in the model. Assumptions 2.12 and 2.13 are new assumptions related to the dynamic condenser system model. ${ }^{[35]}$

\subsection{Reactor Model Equations}

The ODEs in Table 3 are dynamic material balances on chemical species and functional groups, with all concentrations in mmol kg-1 and time in hours. Equation (3.1) is a balance on the total mass $m(\mathrm{~kg})$ of the liquid mixture in the reactor, which decreases over time due to evaporation of water, propanal, monomer, and volatile oligomers (see Assumption 2.4). Masstransfer coefficients $k_{\mathrm{W}}, k_{\mathrm{AD}}$, and $k_{L(i)}$ are in $\mathrm{kg} \mathrm{m}^{-2} \mathrm{~h}^{-1}$ and $A_{\mathrm{b}}$ $\left(\mathrm{m}^{2} \mathrm{~kg}^{-1}\right)$ is the interfacial area of the nitrogen bubbles per unit mass of reactor liquid. [W], $[A D]$, and $[L(i)]$ are concentrations of water, propanal, monomer, and oligomers in the reactor liquid, respectively. $[W]^{*},[A D]^{*}$, and $[L(i)]^{*}$ are corresponding hypothetical concentrations of these species that would be in equilibrium with the corresponding partial pressures in the reactor headspace. Expressions for $[W]^{*}$ and $[L(1)]^{*}$ are provided in Table 4 (i.e., Equations (F1.1) and (F1.2), Supporting Information) whereas $[A D]^{*}$ and $[L(i)]^{*}$ with $i=2$ to 5 are set at zero (Assumption 2.5). $F_{\mathrm{vW}}^{*}$ and $F_{\mathrm{v} L(1)}^{*}$, which appear in Equations (F1.1) and (F1.2) (Supporting Information) are corresponding equilibrium volume fractions of water and monomer in the polymer phase, computed using cubic spline fitting of the VLE data of Xie. ${ }^{[16]}$ The resulting VLE expression used for computing $F_{\mathrm{vW}}^{*}$ from $P_{\mathrm{W}}$ at $180{ }^{\circ} \mathrm{C}$ relies on interpolation between Xie's data obtained at 160 and $185^{\circ} \mathrm{C}$. Because Xie's VLE data for water are only available over a limited range of conditions (see Figure 2a), two additional fictitious data points (i.e., $P_{\mathrm{W}}(0.001)$ and $\left.P_{\mathrm{W}}(0.02)\right)$ are included in the cubic spline fit and appear in the model as adjustable parameters. Using the additional parameters results in improved modeling of the full range of water VLE that is encountered in the dynamic data. Details are provided in the Supporting Information. The monomer cubic-spline VLE expression used for computing $F_{\mathrm{vL} 1}^{*}$ from $P_{L(1)}$ is the same as developed by Cui et al. In Equations (F1.1) and (F1.2) (Supporting Information), $\rho_{\mathrm{W}}, \rho_{L(1)}$, and $\rho$ are densities of water, monomer, and polymer, respectively (in $\mathrm{kg} \mathrm{m}^{-3}$ ) computed using industrial correlations. $M_{\mathrm{W}}, M_{\mathrm{AD}}$, and $M_{L(i)}$ are molar masses of the corresponding species in $\mathrm{kg} \mathrm{mmol}^{-1}$. 
Table 2. Assumptions used to develop the proposed PO3G model.[21-24]
Protonated species do not react with each other due to repulsion between positive charges. Reactions occur only in the liquid phase.
2.2
The exchange of protons between hydroxyl ends and water is sufficiently fast to be in equilibrium. All hydroxyl ends on monomers, oligomers, and on polymer chains have equal probability of being protonated. The probability of any molecule being protonated at more than one location is negligible.
Transetherification between linear chains is sufficiently fast to randomize the chain ends to distribute the unsaturated ends randomly among molecules of different chain lengths.
Water, monomer, propanal, and linear oligomers (dimer to pentamer) are able to evaporate from the liquid to the bubbles. Hexamer and heptamer are less volatile and do not evaporate.
Partial pressures of propanal, dimer, trimer, tetramer, and pentamer in the nitrogen bubbles and head space are sufficiently small to be set at zero in the corresponding mass-transfer expressions.
2.6 Formation and evaporation of cyclic oligomers are neglected in the current model.
2.7 The proportion of protonated water is negligible in comparison with the concentration of neutral water. The proportions of protonated hydroxyl ends, unsaturated ends, and secondary carbocation ends are negligible in comparison with the concentration of neutral hydroxyl ends. The concentration of protonated ether links is small and can be neglected when distributing the protons arising from the super-acid catalyst between hydroxyl ends and water (see Equation (4.6)).
2.8 Protonated secondary hydroxyl ends produced via Reaction (6) are short-lived and do not accumulate in the reactor.
2.9 Propanal is produced primarily from protonated secondary hydroxyl groups on long polymer chains. Generation of propanal from secondary hydroxyl groups on monomers and oligomers (dimers to heptamers) can be neglected.
2.10 Transetherification occurs between protonated hydroxyl ends and neutral ether links.
2.11 The ideal gas law is assumed for the vapor in the reactor head space.
2.12 Because a small amount of monomer evaporates before the catalyst is added to the reactor, the initial liquid in the condenser is pure monomer. The mass of the liquid in the condenser remains constant over time.
Nitrogen in the overhead stream exits the condenser in the vapor. Monomer and linear oligomers that evaporate from the reactor exit the condenser in the liquid. Water and propanal partition between the liquid and vapor phases according to partition coefficient values, which are assumed to be constant and perfectly known.

The updated mass-transfer terms in ODE (3.1) include an adjustable bubble-backmixing parameter $\gamma$, which accounts for uncertainty in the amount of bubble recirculation that occurs. Cui et al. assumed that bubbles are well-mixed within the reactor liquid, corresponding to $\gamma=1.0$ (i.e., Cui assumed that the typical partial pressure of water in the bubbles is equal to the corresponding partial pressure in headspace). Smaller values of $\gamma$ account for the near-zero partial pressures of water and monomer in bubbles that are near to the nitrogen inlet. For example, $\gamma=0.5$ indicates that a typical bubble has water and monomer partial pressures that are half-way between zero and the corresponding headspace partial pressures.

Equation (3.2) is a balance on the super-acid catalyst, with [cat] in mmol kg-1, which changes over time due to the timevarying mass of the liquid in the reactor. Note that, for compactness, $\frac{\mathrm{d} m}{\mathrm{~d} t}$ appears on the right-hand sides of ODE (3.2) to (3.12). When solving the differential equations numerically, the right-hand side of ODE (3.1) was substituted for $\frac{\mathrm{d} m}{\mathrm{dt}}$ in each ODE. ODE (3.3) is a material balance on unreacted monomer, $L(1)$. The first two terms on the right-hand side account for monomer consumption and generation by Reaction (1.2) and its reverse. The factor $f_{1}$ is the fraction of hydroxyl ends in the reaction mixture that are protonated and $f_{2}$ is the fraction of water molecules that are protonated. Factor $f_{\mathrm{E} 1}$ is the fraction of ether links that are next to the end of a linear chain (and hence can produce $L(1)$ via hydrolysis reactions). Expressions for these and other probability factors that appear in the model have been derived and appear in Table 4, along with other algebraic expressions required to solve the model equations. The third and fourth terms in ODE (3.3) account for monomer consumption and generation by Reaction (1.8). The fifth term accounts for evaporation of monomer into the bubbles. The final term accounts for the change in monomer concentration associated with the loss in total mass of the reaction mixture.

Similarly, ODE (3.4) and (3.5) are balances on volatile oligomers (dimer to pentamer), and nonvolatile oligomers (hexamer and heptamer), respectively, in the liquid phase. The factor $f_{E i}$ is fraction of ether links that are $i$ units from an end of a linear chain. This fraction is important because it determines the proportion of hydrolysis (reverse of Reaction (1.2)) and transetherification (Reaction (1.8)) reactions that lead to cleavage of oligomers from the ends of polymer chains.

ODE (3.6) to (3.10) are material balances for water, propanal, hydroxyl ends, unsaturated ends, and secondary carbocation ends, respectively, wherein $f_{3}$ is the fraction of ends that are unsaturated. ODE (3.11) and (3.12) are material balances for the total number of linear molecules $\lambda_{\text {tot }}^{0}$ (i.e, polymer chains,

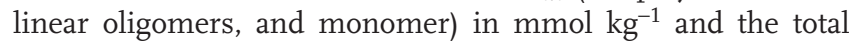
number of (reacted and unreacted) monomer units $\lambda_{\text {tot }}^{1}$, respectively, also in mmol kg-1 ODE (3.13) is a set of material balances for volatile species in the reactor headspace. Partial pressures appear on the left-hand side because the ideal gas law is assumed in the vapor phase (Assumption 2.11). Details concerning the derivation of these ODEs can be found in the Supporting Information. Note that the ODEs in Table 3 and the probability factors in Table 4 (i.e., Equations (4.1) to (4.5)) are considerably simpler than the corresponding ODEs and larger number of probability factors derived by Cui et al. ${ }^{[21-23]}$ due to assumptions 2.7 to 2.11 . The validity of these assumptions, over the range of reactor operating conditions encountered during the experiments, has been confirmed using Cui's model (see the Supporting Information). 
Table 3. Differential equations for PO3G model in reactor.

$$
\frac{\mathrm{d} m}{\mathrm{~d} t}=-m k_{\mathrm{W}} A_{\mathrm{b}}\left([W]-\gamma[W]^{*}\right) M_{W}-m k_{\mathrm{AD}} A_{\mathrm{b}}\left([A D]-\gamma[A D]^{*}\right) M_{\mathrm{AD}}-m \sum_{i=1}^{5} k_{L(i)} A_{\mathrm{b}}\left([L(i)]-\gamma[L(i)]^{*}\right) M_{L(i)}
$$

$\frac{\mathrm{d}[c a t]}{\mathrm{d} t}=-\frac{[c a t]}{m} \frac{\mathrm{d} m}{\mathrm{~d} t}$

$\frac{\mathrm{d}[L(1)]}{\mathrm{d} t}=-4 k_{2} f_{1}[L(1)][O]+k_{2 r} f_{E 1}[E] f_{2}[W]-k_{8} f_{1} 2[L(1)][E]+\frac{1}{2} k_{8} f_{1}[O] f_{E 1}[E]-k_{L(1)} A_{b}\left([L(1)]-\gamma[L(1)]^{*}\right)-\frac{[L(1)]}{m} \frac{\mathrm{d} m}{\mathrm{~d} t}$

$$
\begin{aligned}
\frac{\mathrm{d}[L(i)]}{\mathrm{d} t}= & -4 k_{2} f_{1}[L(i)][O]+k_{2} f_{1} \sum_{k=1}^{i-1} 2[L(k)] 2[L(i-k)]-k_{2 r}(i-1)[L(i)] f_{2}[W]+k_{2 r} f_{E_{\mathrm{Ei}}}[E] f_{2}[W]-k_{8} f_{1} 2[L(i)][E]-k_{8}(i-1)[L(i)] f_{1}[O] \\
& +\frac{1}{2} k_{8} f_{1}[O] f_{E i}[E]+\frac{1}{2} k_{8} \sum_{k=1}^{i-1} f_{1} 2[L(k)] f_{E i-k}[E]-k_{L(i)} A_{b}\left([L(i)]-\gamma[L(i)]^{*}\right)-\frac{[L(i)]}{m} \frac{\mathrm{d} m}{\mathrm{~d} t}
\end{aligned}
$$

where $i=2,3,4,5$

$$
\begin{aligned}
\frac{\mathrm{d}[L(i)]}{\mathrm{d} t}= & -4 k_{2} f_{1}[L(i)][O]+k_{2} f_{1} \sum_{k=1}^{i-1} 2[L(k)] 2[L(i-k)]-k_{2 r}(i-1)[L(i)] f_{2}[W]+k_{2 r} f_{E i}[E] f_{2}[W]-k_{8} f_{1} 2[L(i)][E]-k_{8}(i-1)[L(i)] f_{1}[O] \\
& +\frac{1}{2} k_{8} f_{1}[O] f_{E 1}[E]+\frac{1}{2} k_{8} \sum_{k=1}^{i-1} f_{1} 2[L(k)] f_{E i-k}[E]-\frac{[L(i)]}{m} \frac{\mathrm{d} m}{\mathrm{~d} t}
\end{aligned}
$$

where $i=6,7$

$\frac{\mathrm{d}[\mathrm{W}]}{\mathrm{d} t}=+k_{2} f_{1}[O]^{2}-k_{2 r}[E] f_{2}[W]+k_{3} f_{1}[O]+k_{5} f_{1}[O]-k_{7} K_{6}\left[C_{5}\right][W]-k_{W} A_{b}\left([W]-\gamma[W]^{*}\right)-\frac{[W]}{m} \frac{d m}{d t}$

$\frac{\mathrm{d}[A D]}{\mathrm{d} t}=+k_{7} K_{6}\left[C_{\mathrm{s}}\right][W]-k_{\mathrm{AD}} A_{\mathrm{b}}\left([A D]-\gamma[A D]^{*}\right)-\frac{[A D]}{m} \frac{\mathrm{d} m}{\mathrm{~d} t}$

$\frac{\mathrm{d}[O]}{\mathrm{d} t}=-2 k_{2} f_{1}[O]^{2}+2 k_{2 r}[E] f_{3}[W]-k_{3} f_{1}[O]-k_{5} f_{1}[O]+k_{7} K_{6}\left[C_{s}\right][W]-2 \sum_{i=1}^{5} k_{L(i)} A_{b}\left([L(i)]-\gamma[L(i)]^{*}\right)-\frac{[O]}{m} \frac{d m}{d t}$

$\frac{\mathrm{d}[U]}{\mathrm{d} t}=+k_{3} f_{1}[O]-k_{4}[U] f_{2}[W]-\sum_{i=1}^{5} k_{L u(i)} A_{\mathrm{b}}\left(2 f_{3}[L(i)]-\gamma\left[L_{u}(i)\right]^{*}\right)-\frac{[U]}{m} \frac{\mathrm{d} m}{\mathrm{~d} t}$

$\frac{\mathrm{d}\left[C_{\mathrm{s}}\right]}{\mathrm{d} t}=+k_{4}[U] f_{2}[W]+k_{5} f_{1}[O]-k_{7} K_{6}\left[C_{s}\right][W]-\frac{\left[C_{s}\right]}{m} \frac{\mathrm{d} m}{\mathrm{~d} t}$

$\frac{\mathrm{d} \lambda_{\text {tot }}^{0}}{\mathrm{~d} t}=-k_{2} f_{1}[O]^{2}+k_{2 r}[E] f_{2}[W]+k_{7} K_{6}\left[C_{\mathrm{s}}\right][W]-k_{A D} A_{b}\left([A D]-[A D]^{*}\right)-\sum_{i=1}^{5} k_{L(i)} A_{b}\left([L(i)]-\gamma[L(i)]^{*}\right)-\frac{\lambda_{\text {tot }}^{0}}{m} \frac{\mathrm{d} m}{\mathrm{~d} t}$

$\frac{\mathrm{d} \lambda_{\mathrm{tot}}^{1}}{\mathrm{~d} t}=-k_{\mathrm{AD}} A_{\mathrm{b}}\left([A D]-\gamma[A D]^{*}\right)-\sum_{i=1}^{5} i k_{L(i)} A_{\mathrm{b}}\left([L(i)]-\gamma[L(i)]^{*}\right)-\frac{\lambda_{\mathrm{tot}}^{1}}{m} \frac{\mathrm{d} m}{\mathrm{~d} t}$

$\frac{\mathrm{d} P_{\mathrm{x}}}{\mathrm{d} t}=\left[k_{\mathrm{W}} A_{\mathrm{b}} m\left([x]-\gamma[x]^{\star}\right)-F_{\text {gtot }} \frac{P_{\mathrm{x}}}{P_{\text {tot }}}\right] \frac{R T}{V_{\text {gas }}}$

where $x=W, A D, L(i), i=1,2,3,4,5$

Equation (4.6) shows the relationship between the equilibrium constant $K_{2}$ for the condensation reaction (Reaction (1.2)) and Xie's apparent equilibrium constant $K_{2}$ app, which he measured experimentally at several different temperatures. In Cui's previous models, the parameter $K_{2}$ was inestimable from the kinetic data. In the current model, we treat Xie's apparent equilibrium constant $K_{2}$ app as a relatively well-known parameter, based on his experimental results. Equations (4.7) and (4.8) account for the inhibitory influence of water on the polycondensation rate constant and equilibrium constant, respectively, with the new unknown parameter $K_{\mathrm{H}}$ reducing the condensation rate constant and the equilibrium constant as the water concentration increases.

\subsection{Condenser Model}

The overhead vapor stream from the reactor headspace contains nitrogen, water, propanal, monomer, and volatile oligomers. This stream is fed to the condenser, as shown in Figure 1, which operates at $10{ }^{\circ} \mathrm{C}$ and atmospheric pressure. One of the assumptions in the earlier models of Cui et al. is that the condenser operates as a perfect instantaneous separator with all of the nitrogen exiting in the vapor and other species exiting only in the liquid. ${ }^{[23]}$ Cui's model neglects dynamics due to the liquid hold-up in the condenser and any time delay associated with flow through the overhead piping. ${ }^{[23]}$ She suggested that 
Table 4. Probability factors and algebraic expressions for reactor model.

\begin{tabular}{|c|c|c|}
\hline Expression & Description & \\
\hline$f_{1}=\frac{\left[O_{p}\right]}{[O]}=\frac{f_{2}}{K_{1}}$ & Fraction of hydroxyl ends that are protonated & $(4.1)$ \\
\hline$f_{2}=\frac{\left[W_{p}\right]}{[W]}=\frac{[\text { cat }]}{[W]+\frac{[O]}{K_{1}}}$ & $\begin{array}{c}\text { Fraction of water molecules that are } \\
\text { protonated }\end{array}$ & $(4.2)$ \\
\hline$f_{3}=\frac{[U]}{[O]}$ & Fraction of ends that are unsaturated & $(4.3)$ \\
\hline$f_{E l}=2 \frac{\lambda_{\text {tot }}^{0}-\sum_{j=1}^{i}[L(j)]}{[E]}$ & $\begin{array}{c}\text { Fraction of ether links that are } i \text { units from } \\
\text { an end of a linear chain }\end{array}$ & $(4.4)$ \\
\hline$[E]=\lambda_{\text {tot }}^{1}-\lambda_{\text {tot }}^{0}$ & Concentration of ether links & $(4.5)$ \\
\hline$K_{2}=\frac{K_{2 \text { app }}}{K_{1}}$ & $\begin{array}{l}\text { Equilibrium constant for condensation } \\
\text { reaction }\end{array}$ & $(4.6)$ \\
\hline$k_{2}=\frac{k_{2 \text { reg }}}{1+K_{H}[W]}$ & $\begin{array}{l}\text { Condensation rate constant accounted for } \\
\text { inhibitory effect of water }\end{array}$ & $(4.7)$ \\
\hline$K_{2}=\frac{K_{2 \mathrm{reg}}}{1+K_{\mathrm{H}}[W]}$ & $\begin{array}{l}\text { Condensation equilibrium accounted for } \\
\text { inhibitory effect of water }\end{array}$ & $(4.8)$ \\
\hline
\end{tabular}

the neglected condenser dynamics could be an important cause of the relatively poor fit to oligomer concentration data. ${ }^{[23]}$ The current model relaxes Cui's assumptions by treating the condenser system as an equilibrium flash unit with first-order dynamics and time delay.

Equilibrium flash calculations performed using Aspen HYSYS confirm that essentially all of the nitrogen remains in the vapor stream, and that nearly all of the monomer and linear oligomers condense into the liquid phase. ${ }^{[28]}$ However, water and propanal partition into both phases. This partitioning is accounted for in the current model using partition coefficients $K_{\mathrm{W}}$ and $K_{\mathrm{AD}}$, which are assumed to be constant at $K_{\mathrm{W}}=$ 0.01204 and $K_{\mathrm{AD}}=1.8518$ at $10{ }^{\circ} \mathrm{C}$ and atmospheric pressure (see Assumption 2.13).

Two parameters appear in the extended model to account for the condenser dynamics: the mass of liquid accumulated in the condenser $m_{\mathrm{L}_{\mathrm{c}}}(\mathrm{kg})$ and the time delay $\theta_{\mathrm{d}}(h)$ associated with flow through the overhead piping (i.e., from the reactor vessel to the condenser and from the condenser to the condensate accumulator). $m_{\mathrm{L}_{\mathrm{c}}}$ appears in the ODEs in Table 5, which describe the time-varying concentrations of water $[W]_{\mathrm{C}}$, propanal $[A D]_{\mathrm{C}}$, monomer $[L(1)]_{\mathrm{c}}$, and linear oligomers $[L(i)]_{\mathrm{c}}, i=2 \ldots 5$ in the liquid in the condenser. The condenser system is modeled as if it were an instantaneous flash unit with the resulting liquid fed to a well-mixed tank, as shown in Figure 3. The first term on the right-hand side of ODE (5.1), $F_{\text {gtot }} L_{\mathrm{f}} x_{\mathrm{W}}$, is the flow rate of water from the instantaneous flash into the condenser liquid, where $F_{\text {gtot }}$ is the total molar flowrate of overhead vapor from the reactor, $L_{f}$ is the fraction of this vapor that condenses in the flash, and $x_{\mathrm{w}}$ is the mole fraction of water in this liquid stream. Note that the flow rate $F_{\text {gtot }}$ can be calculated using Equations (6.1) and (6.2) because the pressure in the headspace is constant (i.e., the total molar inflow rate to the headspace is equal to the
Table 5. Differential equations for condenser and condensate collector.

$$
\begin{aligned}
& \frac{\mathrm{d}\left[W_{\mathrm{c}}\right]}{\mathrm{d} t}=\left(F_{\mathrm{gtot}} L_{\mathrm{f}} x_{\mathrm{W}}-F_{\mathrm{gtot}} L_{\mathrm{f}} M_{L_{\mathrm{f}}}\left[W_{\mathrm{c}}\right]\right) \frac{1}{m_{\mathrm{Lc}}} \\
& \frac{\mathrm{d}\left[A D_{\mathrm{c}}\right]}{\mathrm{d} t}=\left(F_{\mathrm{gtot}} L_{\mathrm{f}} x_{\mathrm{AD}}-F_{\mathrm{gtot}} L_{\mathrm{f}} M_{L_{\mathrm{f}}}\left[A D_{\mathrm{c}}\right]\right) \frac{1}{m_{\mathrm{Lc}}}
\end{aligned}
$$

$\frac{\mathrm{d}\left[L(i)_{\mathrm{c}}\right]}{\mathrm{d} t}=\left(F_{\mathrm{gtot}} L_{\mathrm{f}} x_{L(i)}-F_{\mathrm{gtot}} L_{\mathrm{f}} M_{L_{\mathrm{f}}}\left[L(i)_{\mathrm{c}}\right]\right) \frac{1}{m_{\mathrm{Lc}}}$

where $i=1,2,3,4,5$

$\frac{\mathrm{d} m_{\mathrm{cc}}}{\mathrm{d} t}=F_{\mathrm{gtot}} L_{\mathrm{f}} M_{\mathrm{L}_{\mathrm{f}}}$

total outflow rate). Both $L_{\mathrm{f}}$ and $x_{\mathrm{w}}$ (along with the other liquid mole fractions) are obtained via the flash Equations (6.3) to (6.7) in Table 6. Derivations of Equations (6.3) to (6.7) are provided in the Supporting Information. The second term on the right-hand side of ODE (5.1), $F_{\mathrm{gtot}} L_{\mathrm{f}} M_{\mathrm{L}_{\mathrm{f}}}\left[W_{\mathrm{c}}\right]$, is the flow rate of water exiting the condenser before entering the condensate collector. $M_{\mathrm{Lf}_{\mathrm{f}}}$ is the average molecular weight of the liquid flowing from the flash into the condenser, which is obtained using Equation (6.8).

The ODEs in Table 5 are solved along with algebraic Equations (6.1) to (6.8). Equations (6.9) and (6.10) are used to account for the time delay in concentrations of species entering the condensate collector and in $m_{\mathrm{cc}}(\mathrm{kg})$, the mass of the liquid in the condensate collector. The subscript $d$ indicates a delayed state variable. Parameters $\theta_{\mathrm{d}}$ and $m_{\mathrm{Lc}}$ are estimated from the experimental data along with the kinetic, equilibrium, and mass-transfer parameters that appear in the reactor model in Tables 3 and 4.

\subsection{Modeling the Inhibitory Influence of Water on Polycondensation Kinetics and Equilibrium}

Previous models by Mueller et al. and Cui et al. assumed that the polycondensation rate constant and equilibrium constant are independent of water. ${ }^{[19-23]}$ The proposed model accounts for hydration of hydroxyl ends and for polycondensation reactions involving hydrated ends as shown in Table 7. Together these side reactions account for the inhibitory influence of water on polycondensation.

As shown in Reaction (7.1), hydrated ends $O_{\mathrm{H}}$ are assumed to be in fast equilibrium with regular unhydrated hydroxyl ends $O_{\text {reg. }}$ Reaction (7.2) accounts for the reduced reactivity of these hydrated ends compared with regular hydroxyl ends (i.e., $k_{2 \mathrm{H}}<$

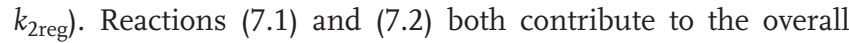
rate of polycondensation, leading to

$r_{2}=k_{2}[O]\left[O_{\mathrm{p}}\right]=k_{2 \mathrm{reg}}\left[O_{\mathrm{reg}}\right]\left[O_{\mathrm{p}}\right]+k_{2 \mathrm{H}}\left[O_{\mathrm{H}}\right]\left[O_{\mathrm{p}}\right]$

where $[O]=\left[O_{\mathrm{reg}}\right]+\left[\mathrm{O}_{\mathrm{H}}\right]$ is the total concentration of regular and hydrated hydroxyl ends. Equation (4) can be rearranged to solve for the overall polycondensation rate constant

$k_{2}=\frac{k_{2 \mathrm{reg}}\left[O_{\mathrm{reg}}\right]+k_{2 \mathrm{H}}\left[O_{\mathrm{H}}\right]}{[\mathrm{O}]}=\frac{k_{2 \mathrm{reg}}\left[O_{\mathrm{reg}}\right]+k_{2 \mathrm{H}}\left[O_{\mathrm{H}}\right]}{\left[O_{\mathrm{reg}}\right]+\left[O_{\mathrm{H}}\right]}$ 


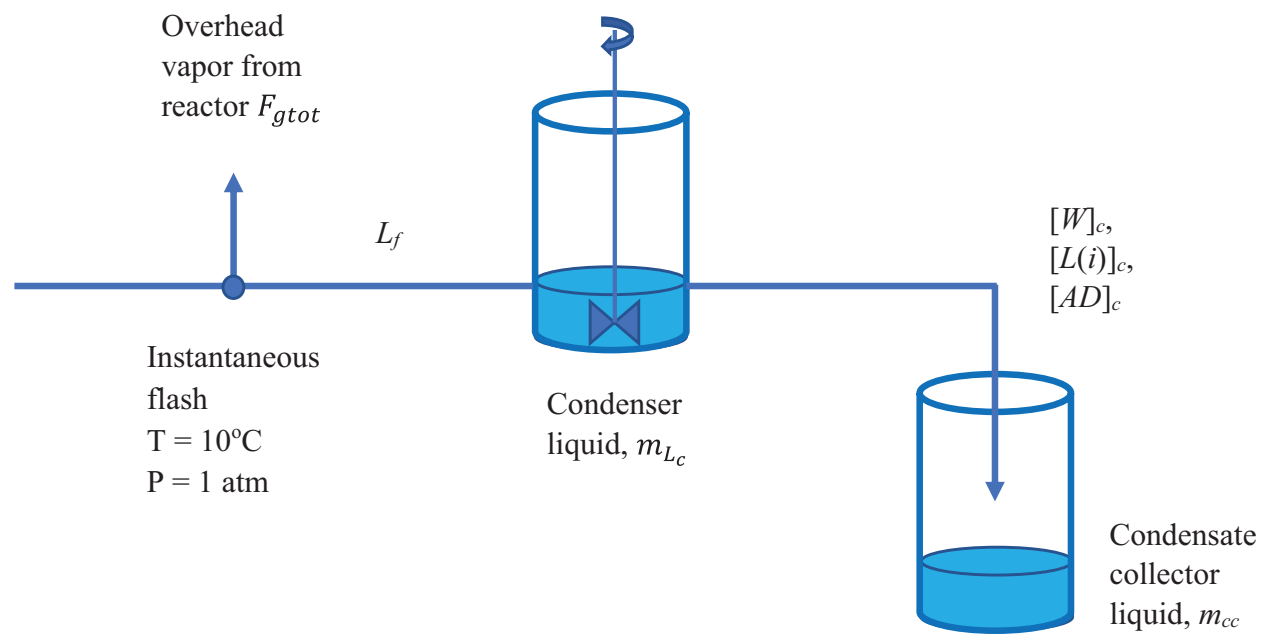

Figure 3. Schematic process for condenser model.

Substituting the equilibrium expression $\left[\mathrm{O}_{\mathrm{H}}\right]=K_{\mathrm{H}}\left[\mathrm{O}_{\mathrm{reg}}\right][\mathrm{W}]$ into Equation (5) gives

$k_{2}=\frac{k_{2 \mathrm{reg}}\left[O_{\mathrm{reg}}\right]+k_{2 \mathrm{H}} K_{\mathrm{H}}\left[O_{\mathrm{reg}}\right][\mathrm{W}]}{\left[O_{\mathrm{reg}}\right]+K_{\mathrm{H}}\left[O_{\mathrm{reg}}\right][\mathrm{W}]}=\frac{k_{2 \mathrm{reg}}+k_{2 \mathrm{H}} K_{\mathrm{H}}[\mathrm{W}]}{1+K_{\mathrm{H}}[\mathrm{W}]}$

Assuming that $k_{2 \mathrm{H}} K_{\mathrm{H}}[\mathrm{W}] \ll k_{2 \text { reg }}$, the overall rate constant becomes $k_{2}=\frac{k_{2 \mathrm{reg}}}{1+K_{\mathrm{H}}[\mathrm{W}]}$ which is Equation (4.7) in Table 4. Note that this water-dependent rate constant has the same type

Table 6. Algebraic expressions for condenser model.

$\left.F_{\mathrm{gx}}=m k_{\mathrm{X}} A_{\mathrm{b}}([X]-\gamma X]^{*}\right)$

where $x$ is $W, A D$, and $L(i)$ with $i=1,2,3,4,5$

$F_{\mathrm{gtot}}=F_{\mathrm{W}}+F_{\mathrm{AD}}+\sum_{i=1}^{5} F_{\mathrm{gL}(i)}+F_{\mathrm{gN}_{2}}$

$z_{\mathrm{x}}=\frac{F_{\mathrm{gx}}}{F_{\mathrm{gtot}}}$

where $x$ is $W, A D$, and $L(i)$ with $i=1,2,3,4,5$

$L_{f}=\frac{z_{W} L_{f}}{K_{W}+L_{f}\left(1-K_{W}\right)}+\frac{z_{A D} L_{f}}{K_{A D}+L_{f}\left(1-K_{A D}\right)}+z_{\sum L(i)}$

$x_{W}=\frac{z_{W}}{K_{W}+L_{f}\left(1-K_{W}\right)}$

$x_{\mathrm{AD}}=\frac{z_{\mathrm{AD}}}{K_{\mathrm{AD}}+L_{f}\left(1-K_{\mathrm{AD}}\right)}$

$x_{\sum L(i)}=\frac{z \sum L(i)}{L_{f}}(i=1 \ldots 5)$

$M_{\mathrm{L}_{\mathrm{f}}}=x_{\mathrm{W}} M_{\mathrm{W}}+x_{\mathrm{AD}} M_{\mathrm{AD}}+x_{\sum L(i)} M_{\sum L(i)}$

where $i=1 \ldots 5$

$\left[X_{\mathrm{cd}}\right](t)=\left[X_{\mathrm{c}}\right]\left(t-\theta_{\mathrm{d}}\right)$

where $X$ is $W, A D$, and $L(i)$ with $i=1 \ldots 5$

$m_{\mathrm{ccd}}(t)=m_{\mathrm{cc}}\left(t-\theta_{\mathrm{d}}\right)$ of water-dependence as Equation (1) used by Okamoto et al. for esterification reactions involving small molecules.

The overall equilibrium constant for polycondensation is

$K_{2}=\frac{[E]\left[W_{\mathrm{p}}\right]}{\left[O_{\mathrm{p}}\right][O]}$

Substituting $[O]=\left[O_{\text {reg }}\right]+\left[O_{\mathrm{H}}\right]$ and $\left[O_{\mathrm{H}}\right]=K_{\mathrm{H}}\left[O_{\text {reg }}\right][\mathrm{W}]$ into Equation (7) gives

$K_{2}=\frac{[E]\left[W_{\mathrm{p}}\right]}{\left[O_{\mathrm{p}}\right]\left(\left[O_{\text {reg }}\right]+K_{\mathrm{H}}\left[O_{\text {reg }}\right][W]\right)}=\frac{[E]\left[W_{\mathrm{p}}\right]}{\left[O_{\mathrm{p}}\right]\left[O_{\text {reg }}\right]\left(1+K_{\mathrm{H}}[W]\right)}$

Substituting $K_{2 \text { reg }}$ for $\frac{[E]\left[W_{p}\right]}{\left[O_{p}\right]\left[O_{\text {reg }}\right]}$ in Equation (8) gives $K_{2}=\frac{K_{2 \text { reg }}}{1+K_{\mathrm{H}}[W]}$ which is Equation (4.12) in Table 4. Estimation of $k_{2 \text { reg }}$ and $K_{2 \text { reg }}$ (instead of $k_{2}$ and $K_{2}$ ) and including the additional parameter $K_{\mathrm{H}}$ result in a proposed model with 21 parameters.

\section{Parameter Estimation and Simulation Results}

The following objective function was used for parameter estimation

$$
\begin{aligned}
J= & \frac{1}{s_{\gamma p p m}^{2}} \sum\left([L(1)]_{m}-[L(1)]\right)^{2}+\frac{1}{s_{\gamma p p m}^{2}} \sum\left([L(i)]_{m}-[L(i)]\right)^{2} \\
& +\frac{1}{s_{w}^{2}} \sum\left([w]_{m}-[w]\right)^{2}+\frac{1}{s_{\gamma p p m}^{2}} \sum\left([A D]_{m}-[A D]\right)^{2} \\
& +\frac{1}{s_{\gamma U}^{2}} \sum\left([U]_{m}-[U]\right)^{2}+\frac{1}{s_{\gamma c p p m}^{2}} \sum\left(\left[L(1)_{c d}\right]_{m}-\left[L(1)_{c d}\right]\right)^{2} \\
& +\frac{1}{s_{\gamma c p p m}^{2}} \sum\left(\left[L(i)_{c d}\right]_{m}-\left[L(i)_{c d}\right]\right)^{2}+\frac{1}{s_{W c}^{2}} \sum\left(\left[w_{c d}\right]_{m}-\left[W_{c d}\right]\right)^{2} \\
& +\frac{1}{s_{\gamma c p p m}^{2}} \sum\left(\left[A D_{c d}\right]_{m}-\left[A D_{c d}\right]\right)^{2}+\frac{1}{s_{W_{c c}}^{2}} \sum\left(m_{c c d m}-m_{c c d}\right)^{2}
\end{aligned}
$$


Table 7. Side reactions that influence polycondensation kinetics and equilibrium.

$$
\begin{aligned}
& \mathrm{O}_{\mathrm{reg}}+W \stackrel{K_{H}}{\rightleftharpoons} O_{H} \\
& O_{\mathrm{p}}+O_{\mathrm{H}} \underset{k_{2 H \mathrm{r}}}{\stackrel{k_{2 H}}{\rightleftharpoons}} E+W_{\mathrm{p}}+W \\
& O_{\mathrm{p}}+O_{\text {reg }} \underset{k_{\text {2ereg }}}{\stackrel{k_{\text {2eg }}}{\rightleftharpoons}} E+W_{p}
\end{aligned}
$$

The first six terms on the right-hand side include the measured concentrations of species in the liquid phase (i.e., monomer $[L(1)]_{\mathrm{m}}$, linear oligomers $[L(i)]_{\mathrm{m}}, i=2$ to 7 , water $[W]_{\mathrm{m}}$, propanal $[A D]_{\mathrm{m}}$, and unsaturated ends $\left.[U]_{\mathrm{m}}\right)$. The next five terms include measured delayed concentrations of the species exiting the condenser (i.e., monomer $\left[L(1)_{\mathrm{cd}}\right]_{\mathrm{m}}$, linear oligomers $\left[L(i)_{\mathrm{cd}}\right]_{\mathrm{m}}, i=2 \ldots .5$, water $\left[W_{\mathrm{cd}}\right]_{\mathrm{m}}$, and propanal $\left.\left[A D_{\mathrm{cd}}\right]_{\mathrm{m}}\right)$. The final term includes the measured mass of liquid in the condensate collector, $m_{\text {ccdm }}$.

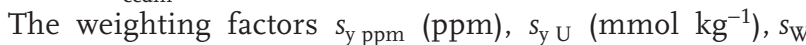
$(\mathrm{wt} \%), s_{\mathrm{yc}} \mathrm{ppm}(\mathrm{ppm}), s_{\mathrm{Wc}}(\mathrm{wt} \%)$, and $s_{\mathrm{m}_{\mathrm{cc}}}(\mathrm{kg})$ account for uncertainties in the various measurements. Note that a common weighting factor $s_{y}$ ppm is used to account for uncertainty in measurements of monomer, oligomers, and propanal in liquid phase. The value shown in Table G1 (Supporting Information) is $5 \%$ of the average measured value for these species over the range of experimental conditions in the data because we have greater trust (in a relative sense) in measurements of species with higher concentrations than those with lower concentrations. Weighting factor $s_{\mathrm{y}} \mathrm{U}$ for unsaturated ends is equal to $s_{\mathrm{y} p m}$, with the units converted to account for reporting of unsaturated ends in $\mathrm{mmol}$ $\mathrm{kg}^{-1}$ rather than ppm. The weighting factor for water in the liquid phase $s_{\mathrm{W}}$ is $5 \%$ of the average measurement of water in the liquid phase. Similarly, $s_{\mathrm{yc}} \mathrm{ppm}$ is set at $5 \%$ of the mean measurement of the species concentrations of monomer, oligomers, and propanal in the condensate. Similarly, $s_{\mathrm{Wc}}$ and $s_{m_{c c}}$ are set at $5 \%$ of the average values of corresponding measurements.

In the current study, parameter estimation is performed using three steps. In the first step, values of the 17 kinetic and mass-transfer parameters in Table 8 are estimated in the simplest revised model, which accounts for condenser dynamics, but uses the prior VLE expressions for water and monomer developed by $\mathrm{Xie}^{[16]}$ and Cui et al., ${ }^{[22]}$ respectively. In this simplest improved model, the bubble backmixing parameter $\gamma$ is set at 1.0 to correspond to the complete backmixing assumption of Cui et al.,[21-23] and the polycondensation kinetic parameter $k_{2}$ and equilibrium parameter $K_{2}$ are assumed to be independent of water. In step 2, the VLE expressions are replaced by Equations (F1.3) and (F1.4) (Supporting Information), with the corresponding parameters $P_{\mathrm{W}}(0.001)$ and $P_{\mathrm{W}}(0.02)$ for the water VLE introduced into the list of parameters. In addition, the adjustable bubblebackmixing parameter is estimated to account for uncertainty in the bubble circulation pattern. In step 3, the model is extended further to account for the inhibitory effect of water, using Equations (4.7) and (4.8), so that $k_{2 \text { reg }}, K_{2 \text { reg }}$, and $K_{\mathrm{H}}$ are
Table 8. Parameter estimation for the improved PO3G model: step 1.

\begin{tabular}{lcccccc}
\hline $\begin{array}{l}\text { Para- } \\
\text { meter }\end{array}$ & Rank & Units & $\begin{array}{c}\text { Initial } \\
\text { value }\end{array}$ & $\begin{array}{c}\text { Lower } \\
\text { bound }\end{array}$ & $\begin{array}{c}\text { Upper } \\
\text { bound }\end{array}$ & Estimate \\
\hline$K_{1}$ & 1 & - & 93 & 1 & $10^{5}$ & 6755.4 \\
$k_{2}$ & 5 & $\mathrm{~kg} \mathrm{mmol}^{-1} \mathrm{~h}^{-1}$ & $8 \times 10^{-3}$ & $8 \times 10^{-4}$ & 0.1 & 0.10 \\
$K_{2} K_{1}$ & 16 & - & 3.17 & 0.1 & 10 & 9.99 \\
$k_{3}$ & 7 & $\mathrm{~h}^{-1}$ & 0.65 & 0 & 1000 & 185.90 \\
$k_{4}$ & 2 & $\mathrm{~kg} \mathrm{mmol}^{-1} \mathrm{~h}^{-1}$ & 0.003 & 0 & 1000 & 562.27 \\
$k_{5}$ & 6 & $\mathrm{~h}^{-1}$ & $1.5 \times 10^{-5}$ & 0 & 1000 & 266.19 \\
$k_{7} K_{6}$ & 12 & $\mathrm{~h}^{-1}$ & 0.02 & 0 & 100 & $6.8 \times 10^{-3}$ \\
$k_{8}$ & 17 & $\mathrm{~kg} \mathrm{mmol}^{-1} \mathrm{~h}^{-1}$ & $10^{-5}$ & $10^{-6}$ & $10^{-4}$ & + \\
$k_{\mathrm{WW}}$ & 3 & $\mathrm{~kg} \mathrm{~m}^{-2} \mathrm{~h}^{-1}$ & 7000 & 0 & $10^{3} \times k_{\mathrm{W} 0}$ & 3409 \\
$k_{\mathrm{AD}}$ & 10 & $\mathrm{~kg} \mathrm{~m}^{-2} \mathrm{~h}^{-1}$ & 1370 & 0 & $10^{3} \times k_{\mathrm{W} 0}$ & $6.95 \times 10^{6}$ \\
$k_{L(1)}$ & 4 & $\mathrm{~kg} \mathrm{~m}^{-2} \mathrm{~h}^{-1}$ & 22.5 & 0 & $10^{3} \times k_{L(1) 0}$ & 12.03 \\
$k_{L(2)}$ & 9 & $\mathrm{~kg} \mathrm{~m}^{-2} \mathrm{~h}^{-1}$ & 0.75 & 0 & $10 \times k_{L(2) 0}$ & 2.10 \\
$k_{L(3)}$ & 11 & $\mathrm{~kg} \mathrm{~m}^{-2} \mathrm{~h}^{-1}$ & 0.25 & 0 & $10 \times k_{L(3) 0}$ & 1.11 \\
$k_{L(4)}$ & 13 & $\mathrm{~kg} \mathrm{~m}^{-2} \mathrm{~h}^{-1}$ & 0.1125 & 0 & $10 \times k_{L(4) 0}$ & 1.12 \\
$k_{L(5)}$ & 15 & $\mathrm{~kg} \mathrm{~m}^{-2} \mathrm{~h}^{-1}$ & 0.06 & 0 & $10 \times k_{L(5) 0}$ & 0.59 \\
$m_{\mathrm{LC}}$ & 8 & $\mathrm{~kg}$ & 0.15 & 0.001 & 1.5 & 0.30 \\
$\theta_{\mathrm{d}}$ & 14 & $\mathrm{~h}$ & 0.2 & 0 & 0.5 & 0.00 \\
\hline & & & & & & \\
\hline
\end{tabular}

estimated instead of $k_{2}$ and $K_{2}$. In all three steps, the model parameters are optimized using a total of 682 data values obtained from four dynamic experiments conducted using different catalyst levels. The optimization is conducted using the Lsqnonlin optimizer in MATLAB with relative and absolute tolerance settings at $10^{-6}$ for the first and second steps and at $10^{-6}$ and $10^{-8}$, respectively, for the third step. These settings were adjusted by trial-and-error to ensure convergence of the objective function and parameter estimates to precise values.

Table 8 shows initial guesses (and lower and upper bounds) for the 17 kinetic parameters considered in step 1 of the estimation process, obtained from the converged estimates of Cui et al. ${ }^{[23]}$ The upper bounds were set to reflect our prior uncertainty in the parameter values, with the upper bounds for poorly known parameters (e.g., $k_{W}$ ) set at 1000 times the corresponding initial guesses. The parameters were ranked from most estimable to the least estimable using an orthogonalization-based parameter-ranking tool. ${ }^{[24,25]}$ The top 16 parameters from the ranked list were selected using Wu's mean-squared-error-based criterion ${ }^{[26,27]}$ to obtain reliable model predictions. The remaining parameter $k_{8}$, which is the transetherification coefficient, was not selected for estimation and was held fixed at its initial guesses. As shown in Table 8 , the additional parameters $m_{\mathrm{Lc}}$ and $\theta_{\mathrm{d}}$ that account for the condenser dynamics and delay are estimable. The estimate for $m_{\mathrm{Lc}}$ (ranked 8th), which accounts for the mass of the liquid hold-up in the condenser is a physically reasonable value of $0.29 \mathrm{~kg}$. The time delay $\theta_{\mathrm{d}}$ (ranked 14th) converges to its lower bound at zero, indicating that delay due to transportation in piping is small. The objective function from step 1 is $J_{1}=5.776 \times 10^{3}$, which is a $94 \%$ improvement compared 
Table 9. Parameter estimation for the improved PO3G model: steps 2 and 3 .

\begin{tabular}{|c|c|c|c|c|c|c|c|}
\hline Parameter & Rank & Unit & Initial value ${ }^{[23]}$ & Lower bound & Upper bound & Estimates from step 2 & Estimates from step 3 \\
\hline$K_{1}$ & 3 & - & 93 & 1 & $10^{5}$ & 4563 & 1.00 \\
\hline$k_{\text {reg }}$ & 6 & $\mathrm{~kg} \mathrm{mmol}^{-1} \mathrm{~h}^{-1}$ & $8 \times 10^{-3}$ & $8 \times 10^{-4}$ & 0.1 & & 0.068 \\
\hline$K_{2 \text { reg }} K_{1}$ & 18 & - & 5 & 0.1 & 10 & & 2.67 \\
\hline$k_{3}$ & 8 & $h^{-1}$ & 0.65 & 0 & 1000 & 669.24 & 0.31 \\
\hline$k_{4}$ & 1 & $\mathrm{~kg} \mathrm{mmol}^{-1} \mathrm{~h}^{-1}$ & 0.003 & 0 & 1000 & 0.061 & 0.80 \\
\hline$k_{5}$ & 5 & $h^{-1}$ & $1.5 \times 10^{-5}$ & 0 & 1000 & $1.8 \times 10^{-3}$ & 0.00 \\
\hline$k_{7} K_{6}$ & 13 & $h^{-1}$ & 0.02 & 0 & 200 & $2.5 \times 10^{-4}$ & 151.38 \\
\hline$k_{8}$ & 19 & $\mathrm{~kg} \mathrm{mmol}^{-1} \mathrm{~h}^{-1}$ & $10^{-5}$ & $10^{-6}$ & $10^{-4}$ & + & + \\
\hline$k_{\mathrm{w}}$ & 2 & $\mathrm{~kg} \mathrm{~m}^{-2} \mathrm{~h}^{-1}$ & 7000 & 0 & $10^{3} \times k_{\mathrm{w}}$ & 1644 & 1749.80 \\
\hline$k_{\mathrm{AD}}$ & 11 & $\mathrm{~kg} \mathrm{~m}^{-2} \mathrm{~h}^{-1}$ & 1370 & 0 & $10^{3} \times k_{W} 。$ & 255.5 & $6.96 \times 10^{6}$ \\
\hline$k_{L(1)}$ & 4 & $\mathrm{~kg} \mathrm{~m}^{-2} \mathrm{~h}^{-1}$ & 22.5 & 0 & $10^{3} \times k_{L(1) \circ}$ & 9.03 & 16.22 \\
\hline$k_{L(2)}$ & 10 & $\mathrm{~kg} \mathrm{~m}^{-2} \mathrm{~h}^{-1}$ & 0.75 & 0 & $10 \times k_{L(2) \circ}$ & 2.00 & 1.67 \\
\hline$k_{L(3)}$ & 12 & $\mathrm{~kg} \mathrm{~m}^{-2} \mathrm{~h}^{-1}$ & 0.25 & 0 & $10 \times k_{L(3) 0}$ & 0.87 & 0.57 \\
\hline$k_{L(4)}$ & 16 & $\mathrm{~kg} \mathrm{~m}^{-2} \mathrm{~h}^{-1}$ & 0.1125 & 0 & $10 \times k_{L(4) \circ}$ & 1.05 & 0.57 \\
\hline$k_{L(5)}$ & 17 & $\mathrm{~kg} \mathrm{~m}^{-2} \mathrm{~h}^{-1}$ & 0.06 & 0 & $10 \times k_{L(5) \circ}$ & 0.59 & 0.39 \\
\hline$m_{\mathrm{Lc}}$ & 9 & $\mathrm{~kg}$ & 0.15 & 0.001 & 1.5 & 0.36 & 0.10 \\
\hline$\theta_{\mathrm{d}}$ & $n / a$ & $\mathrm{~h}$ & 0.2 & 0 & 0.5 & 0.00 & \\
\hline$P_{\mathrm{w}}(0.02)$ & - & $\mathrm{mmHg}$ & 500 & 388.83 & 8151.67 & + & + \\
\hline$P_{\mathrm{W}}(0.001)$ & 15 & $\mathrm{mmHg}$ & 250 & 10 & 352.26 & 342.48 & 10.00 \\
\hline$\gamma$ & 14 & - & 0.9 & 0 & 1 & 0.00 & 0.10 \\
\hline$K_{\mathrm{H}}$ & 7 & $\mathrm{mmol}^{-1} \mathrm{~kg}$ & $2 \times 10^{-3}$ & 0 & 1 & $n / a$ & 0.017 \\
\hline$k_{2}$ & & $\mathrm{~kg} \mathrm{mmol}^{-1} \mathrm{~h}^{-1}$ & $8 \times 10^{-3}$ & $8 \times 10^{-4}$ & 0.1 & 0.10 & \\
\hline$K_{2} K_{1}$ & & - & 5 & 0.1 & 10 & 9.99 & \\
\hline
\end{tabular}

to $J_{0}=9.575 \times 10^{4}$ obtained when Cui's parameter values are used in the model equations. The main improvement to the fit is associated with better predictions of the water concentration in the reactor and species concentrations in the condensate (see Figures E1h, E2, E3h, E4, E5h, E6, E7h, and E8, Supporting Information). Unfortunately, the fit to concentrations of monomer and oligomers in the reactor, and to the mass of liquid accumulated in the condensate collector is still not acceptable (see Figures E5 and E7, Supporting Information), especially for the higher-catalyst runs.

Table 9 shows results from steps 2 and 3 of the parameter estimation, with the same methodology used for ranking, selecting, and estimating the parameters. Note that the ranked order shown in Table 9 is for step 3. Parameter $k_{8}$ remained inestimable, because of its small influence on the model predictions. One of the new VLE parameters $P_{\mathrm{W}}(0.02)$ was also inestimable in steps 2 and 3 and remained fixed at its initial value of $500 \mathrm{mmHg}$, presumably because high water partial pressures were not encountered in the experimental runs used for parameter estimation. The other new VLE parameter $P_{\mathrm{W}}(0.001)$ was ranked 15 th in step 3 , indicating its important influence on the model predictions, along with the bubble-backmixing parameter $\gamma$ (ranked 14th in step 3). After step 2, the objective function became $J_{2}=$
$3.450 \times 10^{3}$, which is a $40 \%$ improvement compared to $J_{1}$. A better fit to the data was obtained for all species, but the model still gives poor fit for oligomer concentrations at long reaction times, suggesting that the consumption rate of oligomers is too slow. The time delay parameter $\theta_{\mathrm{d}}$ was at its lower bound, and statistical analysis revealed that $\theta_{\mathrm{d}}$ and $m_{\mathrm{Lc}}$ were highly negatively correlated. Therefore, we removed the time delay parameter $\theta_{\mathrm{d}}$ (and Equation (6.10)) in the model used in step 3. A new parameter $K_{\mathrm{H}}$ was added in step 3 to account for the inhibitory influence of water. During step 3 , the additional parameter $K_{\mathrm{H}}$ ranked 7 th, indicating that water inhibition seems to have an important influence on the polycondensation reaction (Reaction (1.2)). Parameter $k_{5}$, which accounts for the formation of secondary carbocations from protonated hydroxyl ends, converged to zero, suggesting that Reaction (1.5) can be removed from the model in future modeling studies. Step 3 results in $J_{3}=2.834 \times$ $10^{3}$, which is a further $18 \%$ improvement compared to $J_{2}$. Note that the values of some parameter values changed considerably between steps 2 and step 3 (e.g., $K_{1}$ changed from 4563 to its lower-bound value of 1.00). A correlation matrix (See Appendix H, Supporting Information) reveals that the estimate for $K_{1}$ is highly correlated with the new parameter $K_{\mathrm{H}}$, presumably because both parameters account for the 


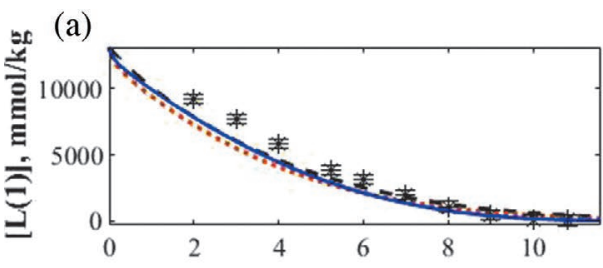

(c)

Time, hr

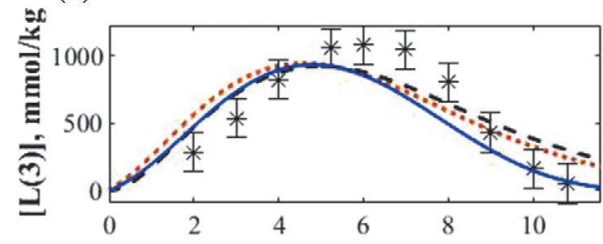

(e)

Time, hr

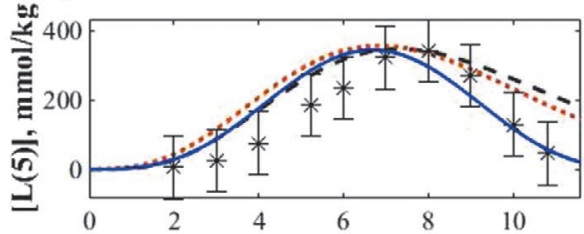

Time, hr

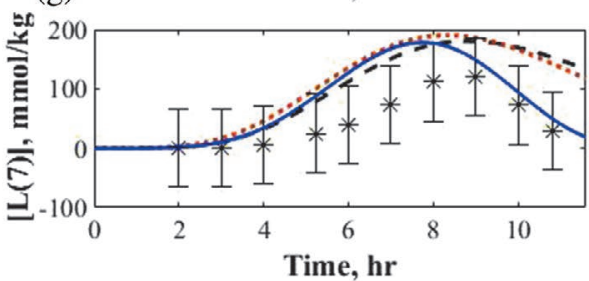

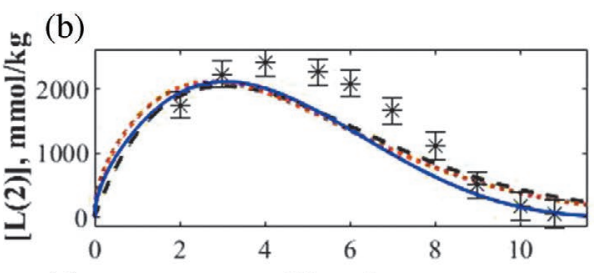

(d) Time, hr

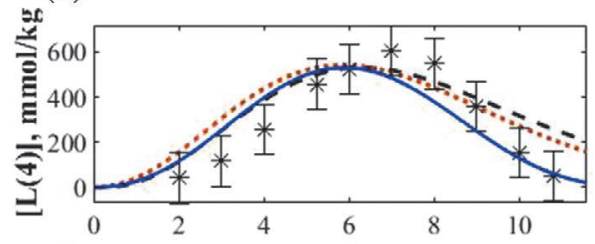

(f)

Time, hr

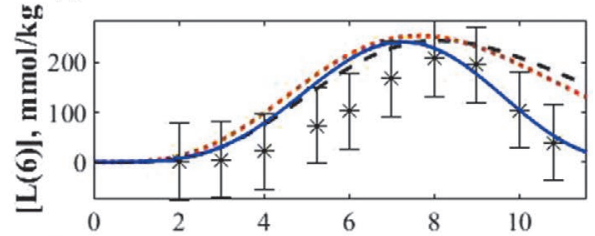

(h)

Time, hr

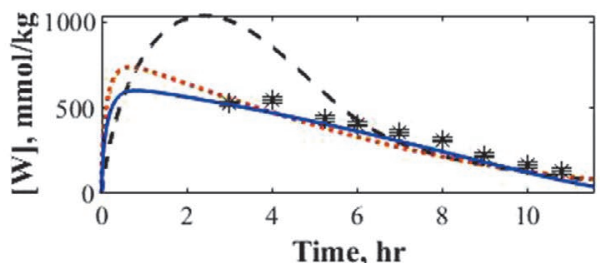

Figure 4. Comparison of model predictions and data obtained using $0.15 \mathrm{wt} \%$ super-acid catalyst: * measured values, predictions from - - step 0 , - step 2, and - step 3 for concentrations of a) monomer, b) dimer, c) trimer, d) tetramer, e) pentamer, f) hexamer, g) heptamer, and h) water in the liquid phase in the reactor.

reduction in the rate of polycondensation at high water levels). Standard deviations for the estimated parameters are also reported in Appendix H (Supporting Information), providing information about which parameter values are statistically different from zero.

Figures 4 and 5 compare the fits for species concentrations in the reactor and in the condensate, respectively, to the measured data $(*)$ obtained from experiments using $0.15 \mathrm{wt} \%$ super-acid catalyst (run 2). This is the second-lowest catalyst level used by Xie in his experiments. ${ }^{[16]}$ Predictions are shown using Cui's parameter estimates (dashed black line), parameter estimates from step 2 (dotted red line), and parameter estimates from step 3 (solid blue line) of the estimation. The error bars show the estimated standard deviation for the measured values. Figures 6 and 7 show similar comparisons for predictions and data obtained using the highest catalyst level (0.25 wt\%, run 4). Similar fits to the data were obtained for two additional runs (with catalyst levels of $0.1 \mathrm{wt} \%$ in run 1 , and 0.20 wt\% in run 3) and results are provided in the Supporting Information. Note that no data were available for the concentration of water in the polymer phase in the lowest-catalyst experiment. Also note that model predictions from step 1 are excluded from Figures 4 to 7 to avoid clutter, and are shown in the Supporting Information.
As shown in Figure 4, the step 3 model and parameter values give the best overall fit to the data obtained using the 0.15 wt\% super-acid catalyst. As shown in Figure 4a, all of the models tend to predict monomer concentrations $[L(1)]$ that are too low at early times (i.e., suggesting that the predicted rate of consumption of $L(1)$ by polycondensation is too high). In Figure 4b-g, the concentrations of the linear oligomers all appear to increase too quickly and reach their maxima at times that are too early, also suggesting that the predicted polycondensation rate is too high. The improved fit obtained using the step 3 model and parameters, compared with steps 1 and 2 (especially at long times) indicates that including the inhibitory influence of water on the polycondensation kinetics and equilibrium is important. The main improvement associated with the revised models in steps 2 and 3 is revealed in Figure $4 \mathrm{~h}$ where Cui's model overpredicts the water concentration at $t<6 \mathrm{~h}$, and the fits obtained from the revised models (in steps 1-3) are considerably better, presumably due to Equations (5.1) to (5.4) for the condenser dynamics.

Figure 5 compares the predictions obtained for species concentrations in the condensate, along with the mass of liquid accumulated in the condensate collector. As shown in Figure $5 \mathrm{a}-\mathrm{g}$, better predictions are obtained from steps 2 and 


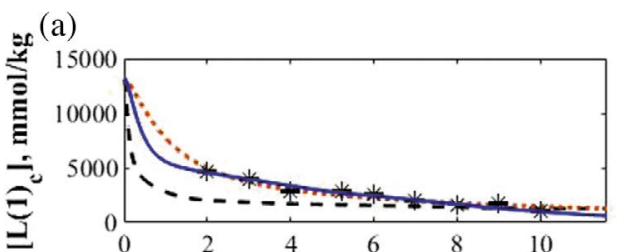

(c)

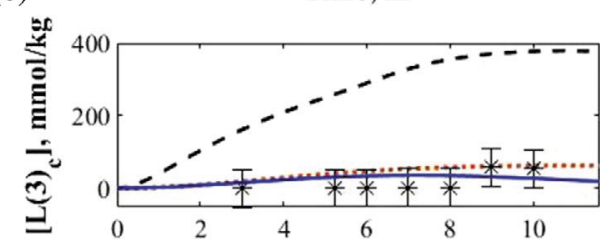

(e)

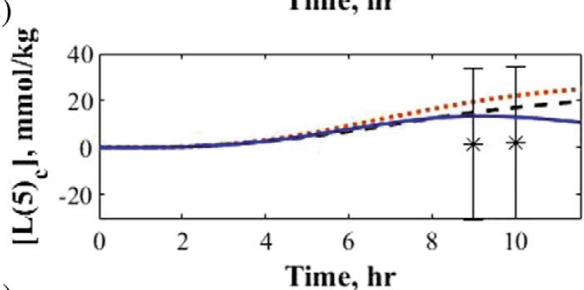

(g)

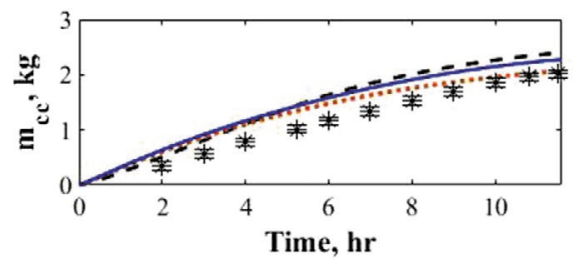

(b)

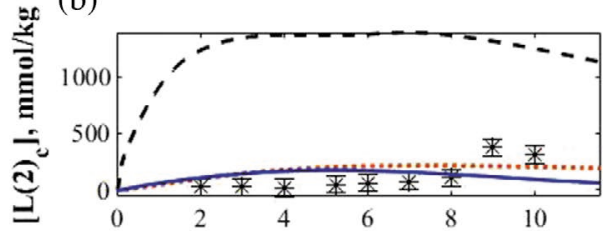

(d)

Time, hr
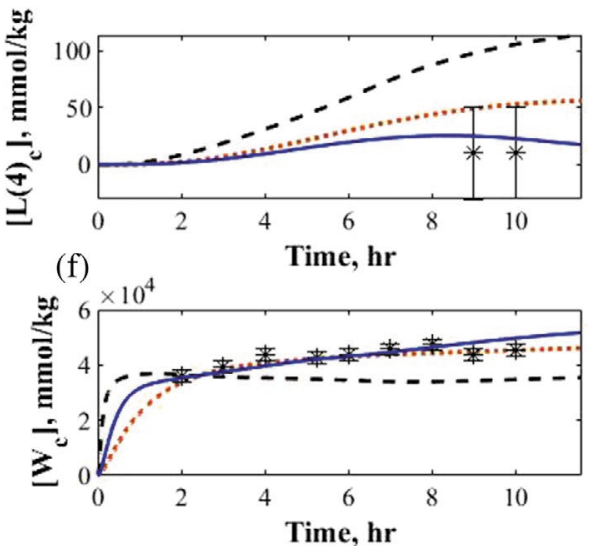

Figure 5. Comparison of model predictions and data obtained using $0.15 \mathrm{wt} \%$ super-acid catalyst: * measured values, predictions from -- step 0 , - step 2, and - step 3 for concentrations of a) monomer, b) dimer, c) trimer, d) tetramer, e) pentamer, and f) water in the condensate exiting the condenser and g) mass of liquid accumulated in the condensate collector.

3 compared to step 0 for concentrations of monomer, oligomer, and water in the condensate, as well as for the mass of the liquid accumulated in the condensate collector. There is very little improvement in fit from step 2 to step 3 in Figure 5 , because including the inhibitory influence of water does not influence the mass-transfer rates and condenser dynamics in an important way.

Figures 6 and 7 show fits to the data obtained at the highest catalyst level (run 4). Similar trends to those in Figure 5 are observed for predictions of monomer and oligomer concentrations in the polymer phase, with the best fit arising from step 3 . The fitted model seems to predict a polycondensation rate that is too high (see Figure $6 \mathrm{a}-\mathrm{g}$ ), where monomer consumption is too fast and the oligomers that are generated are consumed too quickly. The predictions for monomer and oligomers from step 2 are noticeably better than step 1 (see Figures E5 and E7, Supporting Information). The improved fit indicates that updated VLE expression for water and the updated bubble-backmixing parameter $\gamma$ are important for obtaining better predictions of evaporation rates, especially for the high-catalyst runs (runs 3 and 4). The predictions from step 3 are considerably better than the predictions from step 2, suggesting that the inhibitory influence of water plays an important role in the reaction system, especially for obtaining a good fit at long reaction times. As shown in Figure 6h and Figure E7h (Supporting Information), the main reason for reduction in the objective function during the step 1 estimation is the improvement in fit to the water concentration data for the highest-catalyst run. This improvement indicates that including the condenser dynamics results in improved predictions of mass-transfer rates, especially for water. In Figure 7 (and Figures E2, E4, E6, and E8, Supporting Information), the fits to the condensate data are considerably better than those obtained by Cui et al., also indicating that it is important to include the condenser dynamics in the model.

Based on results from three steps of estimation, the most important improvement to the model is including the condenser dynamics (i.e., the biggest percentage reduction of the objective function is from $J_{0}$ to $J_{1}$ ). It is interesting to note that the final model does not include the time delay parameter $\theta_{\mathrm{d}}$ because it is highly negatively correlated with the mass of the liquid accumulated in the condenser $m_{\mathrm{Lc}}$ and has converged to zero during step 2 . The second most important improvement of the model is associated with revising the water VLE expression and releasing Cui's assumption of perfect bubble-backmixing within the reactor vessel. It is interesting that the final estimated VLE parameter $P_{\mathrm{W}}(0.001)$ is at its lower bound. A small value of $P_{\mathrm{W}}(0.001)$ reduces the evaporation rate of water when the concentration of water in 

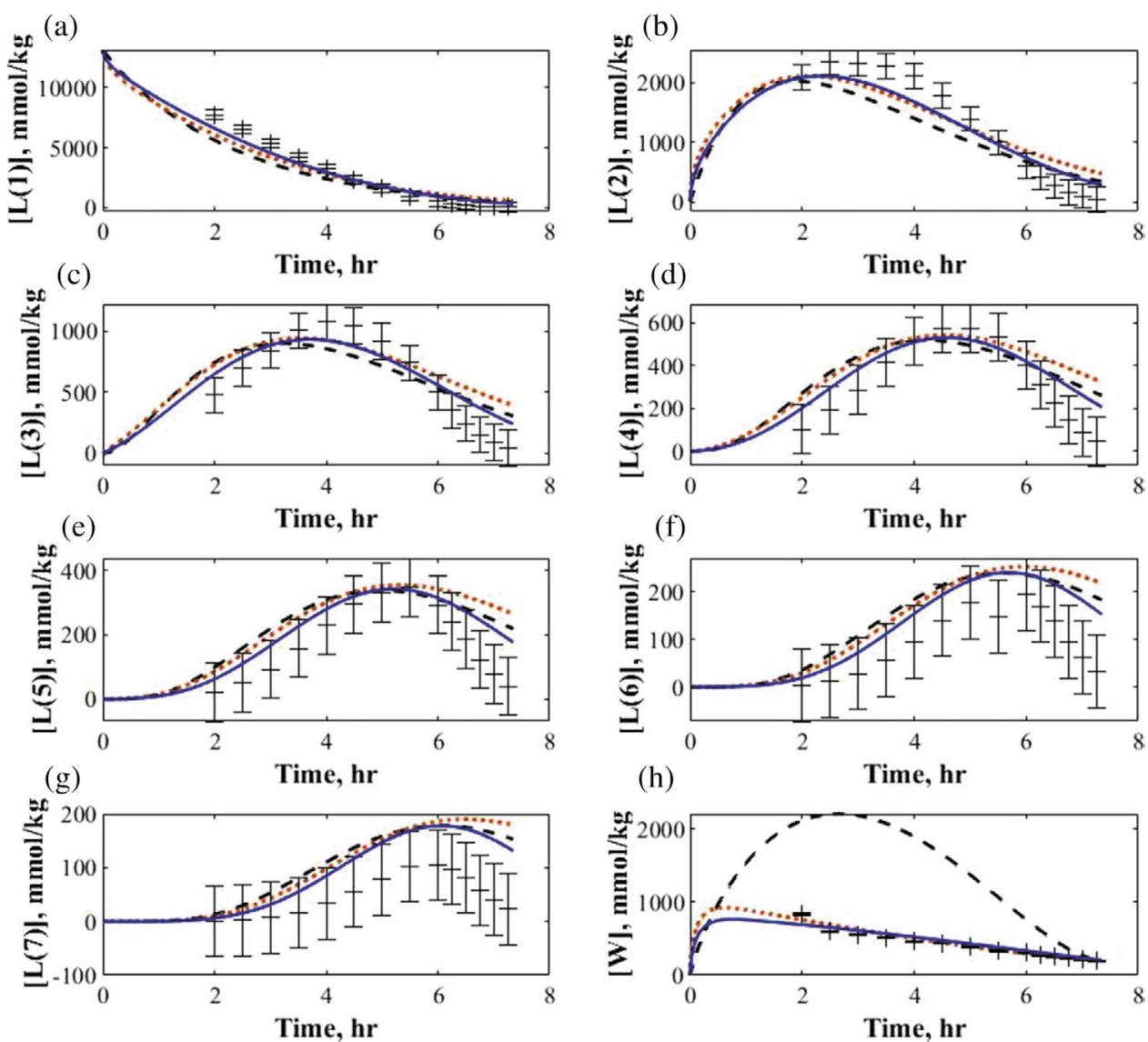

(h)

Time, hr

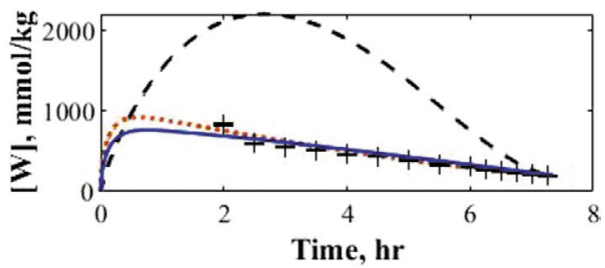

Figure 6. Comparison of model predictions and data obtained using $0.25 \mathrm{wt} \%$ super-acid catalyst: + measured values, predictions from -- step 0 , - step 2, and - step 3 for concentrations of a) monomer, b) dimer, c) trimer, d) tetramer, e) pentamer, f) hexamer, g) heptamer, and h) water in the liquid phase in the reactor.

the polymer phase is small (i.e., at the start and the end of the reaction). More experimental VLE data are required to assess whether the corresponding VLE predictions for water are accurate. Note that parameter $P_{\mathrm{W}}(0.02)$, which was introduced to account for a lack of water VLE data at higher water concentrations, was not selected for estimation. An acceptable fit to the data was obtained using the initial guess of $P_{\mathrm{W}}(0.02)=500 \mathrm{mmHg}$. It is also interesting that $\gamma=0.10$, suggesting that very little backmixing of bubbles occurs. It seems that bubbles near the bottom of the vessel may have considerably lower water and monomer concentrations than bubbles emerging into the headspace at the top surface of the liquid. The third most important improvement to the model is the addition of the inhibitory influence of water. Including this effect improves the fitted oligomer concentrations, especially at long reaction times. Finally, there is still a small discrepancy in the fit to monomer and oligomer concentration data, especially at low reaction times. This lack of fit may be due to the generation and evaporation of cyclic oligomers, which are neglected in the current model. Reactions involving cyclic oligomers (and associated cyclic oligomer concentration data) will be considered in future modeling work. The improved model developed in the current article will provide an important basis for building this more-comprehensive model.

\section{Conclusions}

The current study extends and simplifies the PO3G model developed by Cui et al. to provide a more accurate description of the reactor system and condenser. Probability factors are developed to account for different types of polymer chain ends (i.e., protonated hydroxyl ends, unprotonated hydroxyl ends, and unsaturated ends), which permits considerable simplification of the dynamic material-balance equations. After this initial simplification, the model is extended to account for: i) condenser dynamics and possible deadtime, ii) revised bubble-backmixing and vapor-liquid-equilibrium behavior, and iii) the inhibitory influence of water on polycondensation kinetics. Parameter estimation is conducted in three steps using a parameter subset selection method ${ }^{[26,27]}$ to determine which parameters can and should be estimated from the available industrial data. ${ }^{[16]}$ Parameter estimates from step 1 resulted in a 94\% improvement in the weighted-least-squares objective function, compared to parameter values reported by Cui et al, ${ }^{[23]}$ Incorporation of improved mass-transfer expressions in step 2 further reduced the value of the objective function by $40 \%$ and gave a noticeably better fit to the data, especially for the monomer and oligomer concentrations in the high-catalyst runs. The final step of the estimation, including the inhibitory influence of water, resulted in a further $18 \%$ improvement in the value of the 

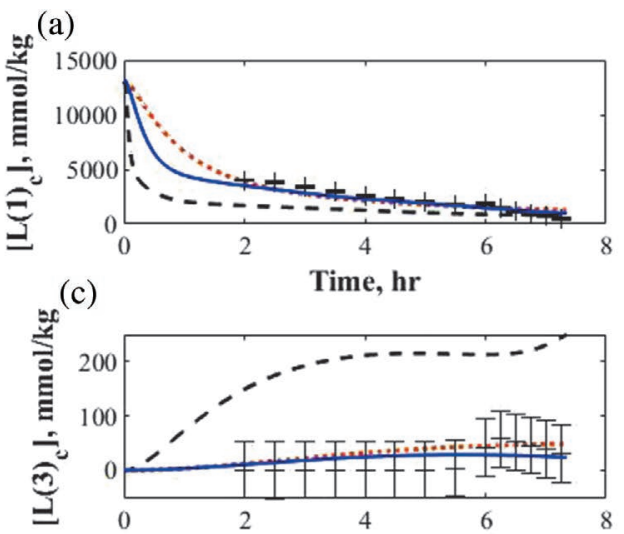

(e)

Time, hr

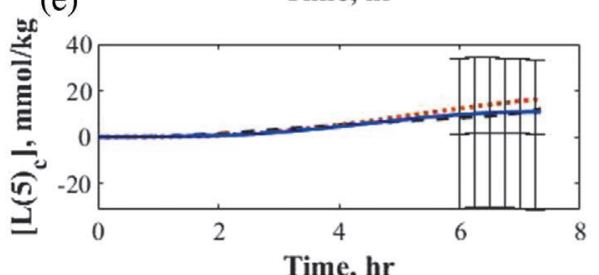

Time, hr

(g)

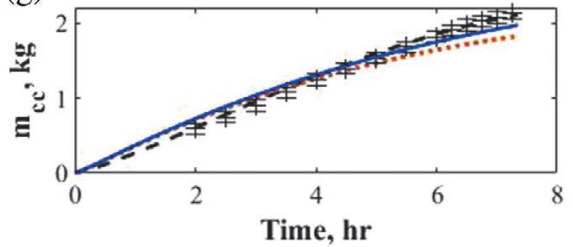

(b)
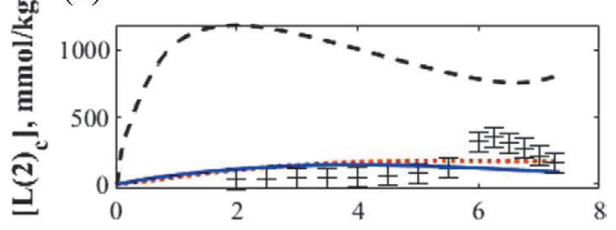

(d)

Time, hr

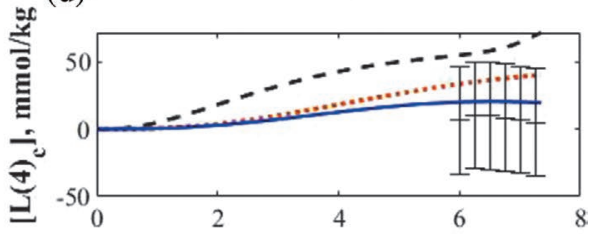

(f)

Time, hr

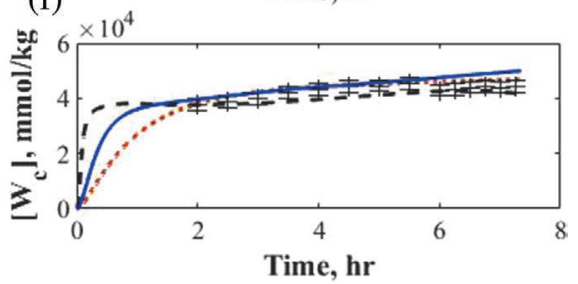

Figure 7. Comparison of model predictions and data obtained using $0.25 \mathrm{wt} \%$ super-acid catalyst: + measured values, predictions from $-\mathbf{-}$ step 0 , ... step 2, and - step 3 for concentrations of a) monomer, b) dimer, c) trimer, d) tetramer, e) pentamer, and f) water in the condensate exiting the condenser and g) mass of liquid accumulated in the condensate collector.

objective function. Although the proposed model is a substantial improvement over previous PO3G models in the literature, there are still some small discrepancies in the dynamic behavior of water, monomer, and oligomer concentrations in the reactor that seem to be associated with an overall polycondensation rate that is too high at low reaction times. In future, a more advanced model will be developed to account for the generation, consumption, and evaporation of cyclic oligomers.

\section{Supporting Information}

Supporting Information is available from the Wiley Online Library or from the author.

\section{Acknowledgements}

The authors acknowledge financial support from Mitacs Globalink and the Natural Sciences and Engineering Research Council of Canada. Data provided by E.I. du Pont Canada are gratefully acknowledged.

\section{Conflict of Interest}

The authors declare no conflict of interest.

\section{Keywords}

mathematical model, polyether, polymerization, propanediol

Received: October 15, 2019

Revised: December 11, 2019

Published online:

[1] H. B. Sunkara, H. R. J. Umile (E. I. du Pont de Nemours and Company), US Patent 6235948, 2001.

[2] H. B. Sunkara, E. K. A. Marchildon, H. C. Ng, L. E. Manzer (E. I. du Pont de Nemours and Company), US Patent 0010374, 2002.

[3] H. B. Sunkara, P. Parmpi (E. I. du Pont de Nemours and Company), US Patent 0283028 A1, 2005.

[4] H. B. Sunkara, L. E. Manzer (E. I. du Pont de Nemours and Company), US Patent 6977291 B2, 2005

[5] H. B. Sunkara, E. K. A. Marchildon, H. C. Ng, L. E. Manzer (E. I. du Pont de Nemours and Company), US Patent 7074968 A2, 2006.

[6] H. B. Sunkara, P. Parmpi (E. I. du Pont de Nemours and Company), US Patent 7074969 B2, 2006.

[7] M. A. Harmer, C. P. Junk (E. I. du Pont de Nemours and Company), WO Patent 041348 A2, 2007.

[8] H. B. Sunkara, H. Q. Do (E. I. du Pont de Nemours and Company), US Patent 7244810 B2, 2007.

[9] H. B. Sunkara, M. Seapan, G. F. Diffendall, F. G. Gallagher (E. I. du Pont de Nemours and Company), US Patent 7342142 B2, 2008. 
[10] M. A. Harmer, C. Hoffmann, S. C. Jackson, E. R. Murphy, R. Spence (E. I. du Pont de Nemours and Company), US Patent 0118464 A1, 2009.

[11] D. C. Confer, M. A. Harmer, C. Hoffmann, S. C. Jackson, S. S. Kristjansdottir, R. Spence (E. I. du Pont de Nemours and Company), US Patent 0118465 A1, 2009.

[12] H. B. Sunkara, H. Q. Do (E. I. du Pont de Nemours and Company), US Patent 7671172 B2, 2010.

[13] A. Minter, E. R. Murphy, H. C. Ng, R. Spence, T. Xie (E. I. du Pont de Nemours and Company), WO Patent 041348, 2011.

[14] H. B. Sunkara, H. Ng, presented to Sarnia Bio-Based Polyols, Sarnia, ON, Canada, October 2006.

[15] H. B. Sunkara, R. W. Miller, presented at 5th Annual World Congress on Industrial Biotechnology a Bioprocessing, Chicago, IL, April 2008.

[16] T. Xie, Polymer Reaction Engineering VII, Niagara Fall, ON, Canada 2009.

[17] R. Hofer, Sustainable Solutions for Modern Economies, Royal Society of Chemistry, Cambridge, UK 2009.

[18] J. V. Kurian, in Natural Fibers, Biopolymers, and Biocomposites (Eds: A. K. Mohanty, M. Misra, L. T. Drzal), Taylor and Francis, Boca Raton, FL 2005, Ch. 15.

[19] P. A. Mueller, E. R. Murphy, B. Rajagopalan, J. P. Congalidis, A. R. Minter, Macromol. Symp. 2011, 302, 56.

[20] P. A. Mueller, B. Rajagopalan, J. P. Congalidis, E. R. Murphy, Macromol. React. Eng. 2012, 6, 126.
[21] W. J. Cui, K. B. McAuley, R. A. Whitney, R. E. Spence, T. Xie, Macromol. React. Eng. 2013, 7, 237.

[22] W. J. Cui, K. B. McAuley, R. E. Spence, T. Xie, Macromol. React. Eng. 2014, 8, 476

[23] W. J. Cui, K. B. McAuley, R. E. Spence, T. Xie, Macromol. React. Eng. 2015, 9, 186

[24] D. E. Thompson, K. B. McAuley, P. J. McLellan, Macromol. React. Eng. 2009, 3, 160.

[25] K. Z. Yao, B. M. Shaw, B. Kou, K. B. McAuley, D. W. Bacon, Polym. React. Eng. 2003, 11, 563.

[26] K. A. P. McLean, S. Wu, K. B. McAuley, Ind. Eng. Chem. Res. 2012, 51,6105

[27] S. Wu, K. B. McAuley, T. J. Harris, Can. J. Chem. Eng. 2011, 89, 325.

[28] A. D. Vo, Ph.D. thesis, Queen's University, Kingston, ON, Canada 2020.

[29] X. Yin, B. Yang, S. Goto, Int. J. Chem. Kinet. 1995, 27, 1065.

[30] B. L. Yang, M. Maeda, S. Goto, Int. J. Chem. Kinet. 1998, 30, 137.

[31] B.-L. Yang, S. B. Yang, R. Yao, React. Funct. Polym. 2000, 44, 167.

[32] K. Okamoto, M. Yamamoto, Y. Otoshi, T. Semoto, M. Yano, K. Tanaka, H. Kita, J. Chem. Eng. Jpn. 1993, 26, 475.

[33] Y. Liu, E. Lotero, J. G. Goodwin, J. Mol. Catal. A: Chem. 2006, 245, 132.

[34] F. F. Liu, K. B. McAuley, Macromol. React. Eng. 2019, 13, 1900037.

[35] A. D. Vo, A. Elraghy, K. B. McAuley, IFAC-PapersOnLine 2018, 51, 661. 